\title{
Systematic review and meta-analysis determining the benefits of in vivo genetic therapy in spinal muscular atrophy rodent models
}

\author{
Ellie M. Chilcott $\mathbb{D}^{1,3 凶}$, Evalyne W. Muiruri ${ }^{1}$, Theodore C. Hirst ${ }^{2}$ and Rafael J. Yáñez-Muñoz $\mathbb{D}^{1 凶}$ \\ (c) The Author(s) 2021, corrected publication 2022
}

\begin{abstract}
Spinal muscular atrophy (SMA) is a severe childhood neuromuscular disease for which two genetic therapies, Nusinersen (Spinraza, an antisense oligonucleotide), and AVXS-101 (Zolgensma, an adeno-associated viral vector of serotype 9 AAV9), have recently been approved. We investigated the pre-clinical development of SMA genetic therapies in rodent models and whether this can predict clinical efficacy. We have performed a systematic review of relevant publications and extracted median survival and details of experimental design. A random effects meta-analysis was used to estimate and compare efficacy. We stratified by experimental design (type of genetic therapy, mouse model, route and time of administration) and sought any evidence of publication bias. 51 publications were identified containing 155 individual comparisons, comprising 2573 animals in total. Genetic therapies prolonged survival in SMA mouse models by 3.23 -fold (95\% Cl 2.75-3.79) compared to controls. Study design characteristics accounted for significant heterogeneity between studies and greatly affected observed median survival ratios. Some evidence of publication bias was found. These data are consistent with the extended average lifespan of Spinraza- and Zolgensma-treated children in the clinic. Together, these results support that SMA has been particularly amenable to genetic therapy approaches and highlight SMA as a trailblazer for therapeutic development.
\end{abstract}

Gene Therapy (2022) 29:498-512; https://doi.org/10.1038/s41434-021-00292-4

\section{INTRODUCTION}

Spinal muscular atrophy (SMA) is a neuromuscular disease chiefly characterised by degenerating alpha motor neurons (MNs) caused by defects in the gene Survival Motor Neuron 1 [1]. SMA is the second most common autosomal recessive disease after Cystic Fibrosis [2] and is also the most common genetic disease resulting in infantile death [3]. MN loss results in atrophy of skeletal muscles, paralysis and denervation of neuromuscular junctions [3]. SMA mostly affects children, with symptoms including muscle weakness, areflexia, difficulty swallowing and feeding, and in the most severe cases is fatal, with infantile death most commonly attributed to respiratory failure [4]. Although MNs are the cells primarily affected in this disease, systemic pathology exists. Muscular [5], vascular [6] and cardiac defects [7] have been reported.

$95 \%$ of SMA patients show deletions of SMN1, with the remaining $5 \%$ carrying mutations in this gene. Homozygous deletions or mutations lead to no SMN protein production from $S M N 1$, however this can be partially compensated for by the duplicated SMN2 gene. Within SMN2, a C to T mutation 6-bp into exon 7 preferentially results in an alternatively spliced transcript lacking exon 7, known as $S M N \Delta 7$, which, when translated, leads to a truncated protein rapidly degraded. SMN2 produces a small amount of full-length transcript and hence protein. The number of SMN2 copies correlates inversely with the severity of SMA [8].
Two SMA treatments, Spinraza and Zolgensma, have been approved for marketing by the US Food and Drug Administration (FDA) and European Medicines Agency (EMA) within the last few years. Spinraza is an antisense oligonucleotide targeting SMN2 splicing, aiming to promote inclusion of exon 7 within transcripts and hence the synthesis of full-length SMN protein. Spinraza binds to SMN2 pre-mRNA at an intronic splicing sequence in intron 7, preventing negative splice factors from binding this site. This causes recognition of exon 7 by U1snRNP and inclusion in the mature SMN2 mRNA transcript [9]. Zolgensma is a selfcomplementary AAV9 vector encoding SMN1. This therapy aims to replace the missing SMN1 gene in SMA patients, thus restoring normal SMN protein function [10]. Both of these therapies were extensively tested in pre-clinical experiments before progressing to clinical trials. The approval of Spinraza was largely underpinned by data from ENDEAR and CHERISH clinical trials, whist only the START clinical trial using Zolgensma was completed prior to licensing. It is important to state that, technically, oligonucleotides are not classed as gene therapies by FDA or Advanced Therapy Medicinal Products by the EMA, while viral vectors like Zolgensma are. We therefore refer to both of them as "genetic" therapies in the current analysis. One more treatment for SMA, Risdiplam (Evrysdi) has been recently approved by both FDA and EMA. It is based on a small molecule able to alter the

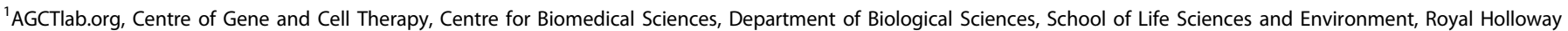

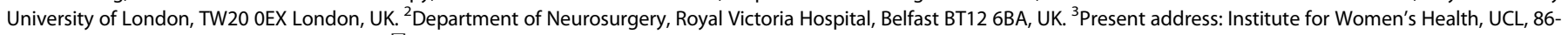
96 Chenies Mews, London WC1E 6HX, UK. ${ }^{凶}$ email: e.crompton@ucl.ac.uk; rafael.yanez@royalholloway.ac.uk 
splicing of SMN2 [11]; not being a genetic therapy, we will not discuss it further.

Here, we review all studies that have used a genetic therapy approach to treat SMA rodent models using meta-analytic techniques to provide quantitative data pertaining to treatment efficacy. This information is useful as it can provide insights into the most successful strategies in pre-clinical research, avoiding unnecessary and unethical repetition of animal experimentation [12], and identify gaps in knowledge that can be addressed in the future. Potential sources of bias and heterogeneity within preclinical studies were also explored. We discuss how effectively preclinical data can predict clinical trial outcome.

\section{DATA SOURCE AND ANALYTICAL METHODS Study identification}

The electronic databases PubMed and Web of Science were searched for relevant published studies between 1950 and 12th June 2020. Keyword strings "gene therapy AND spinal muscular atrophy" and "antisense oligonucleotide AND spinal muscular atrophy" were used. Despite literature often colloquially referring to the use of oligonucleotide-based approaches in gene therapy experiments, oligonucleotides are not officially classed as gene therapies by the FDA, or Advanced Therapy Medicinal Products by EMA. Therefore, the term genetic therapies has been used for the remainder of this analysis, with the exception of the search criteria. All languages were included in the search. No restriction concerning type of publication was used. Manual searching of the bibliographies of each of the electronically identified studies revealed references for additional studies which were then retrieved.

\section{Study selection criteria}

Primary studies found from the electronic and manual searches were screened for eligibility based on the following inclusion criteria [1]: genetic therapy was administered in vivo [2]; a rodent model of SMA was used [3]; median survival data was reported in text, or was calculable form Kaplan-Meier plots; and [4] the number of animals in control and treated groups were reported. Here, in vivo genetic therapy was defined as the introduction of genetic material (DNA, RNA, oligonucleotides, viral vectors, bacterial vectors or genome editing technology) directly into an animal. All studies using pharmacological means to manipulate gene expression, for example histone deacetylase inhibitors or compounds such as Branaplam and Risdiplam, were excluded. No restrictions on the type of SMA rodent model were enforced.

\section{Data extraction of primary studies}

Survival data and aspects of experimental design for each comparison were extracted from included publications. Experimental design characteristics included the type of genetic therapy agent used, rodent model, therapeutic target, delivery route and time of administration. Here, P1 was designated as the day of birth. Disparity was observed in the reporting of viral vector dose, with some studies using the total number of vector genomes $(\mathrm{vg})$ administered per animal and others using vector genomes per kilogram (vg/kg). Here, all doses were converted to $\mathrm{vg} / \mathrm{kg}$ using an approximate birth weight of $1 \mathrm{~g}$ per pup. Outcome data were recorded as median survival (the number of days at which $50 \%$ of animals were alive), and the number of animals in both control and experimental cohorts was recorded. If no median survival data were reported in the text, this was calculated from figures or sought through direct contact with authors. If more than $50 \%$ of animals survived at the end of the reported time period, the median survival value was recorded as the last time point of assessment. If studies presented multiple control groups, the following hierarchy was implemented and data were extracted from that of the highest relevance [1]: reporter gene (if viral vector) or scrambled ASO (if oligonucleotide) [2]; sham surgery or saline injection [3]; untreated. If data were presented from both heterozygous and homozygous SMA animal models, data were extracted from that of homozygous comparisons. If any data were not reported within the study, or if clarification was necessary, study authors were contacted; if no reply was received after two weeks, the relevant studies were excluded.

\section{Data analysis}

Standard meta-analysis techniques could not be employed here given that no standard error or deviation is associated with median survival data. Therefore, the meta-analysis workflow used here was adapted using techniques presented in [12]. This approach has proven successful in other recent pre-clinical meta-analyses [13] and has shown to be comparable to standard (hazard or odds ratio) techniques [14].

Median survival ratios (MSR), equivalent to the survival of treated animals divided by survival of control animals, were calculated to summarise the median survival data that were extracted. This approach was used to maximise consistency with the hazard ratio method commonly used in meta-analyses $[14,15]$. Log-transformed MSRs were entered into a random effects model adapted from DerSimonian and Laird $[16,17]$ with the number of animals used as a measure of precision to weight each study. The number of animals was calculated as the sum of treated and true control (number of control animals divided by the number of treatment groups per control group) animals. To achieve an estimate of variance from data that does not contain an inherent error or deviation value, a fixed effect size with associated measure of heterogeneity, denoted by tau, was first calculated. This was then substituted into the random effects model. Finally, the overall MSR (a measure of whether treatment provided a therapeutic benefit or not) was calculated with associated $95 \%$ confidence intervals and a final random effects standard error. An MSR of 1 represents a neutral treatment effect, $<1$ suggested genetic therapy was detrimental to survival, $>1$ suggests genetic therapy provided a survival advantage.

A stratified meta-analysis was undertaken so that the effect of different experimental intervention conditions could be analysed. The effect of heterogeneity across strata was identified using the $x^{2}$ statistic to determine a threshold level of significance to compare all individual stratifications to. Seven strata were used in this review; type of genetic therapy, dosage of viral vector, overall therapeutic target and SMN1- versus SMN2-based approaches, mouse model and finally route and time of administration. The $x^{2}$ statistic with degrees of freedom equal to the number of substrata minus 1 was adjusted to account for stratifications using the Bonferroni correction. The threshold level of significance calculated was equal to $P=0.0073$. Stratifications that produced a Bonferroni adjusted $P$ value less than $P=0.0073$ suggested that heterogeneity between sub-strata, and thus the MSR, was significantly different from one another.

Publication bias was assessed using funnel plots, Egger regression [18] and Trim and Fill analysis [19] using the number of animals as a measure of precision. The number of animals, instead of inverse variance, as previously described $[12,13]$ avoids potential correlation between standard error and effect sizes that can cause the appearance of funnel plot asymmetry [20].

Software. Searches were uploaded to the Collaborative Approach to Meta-Analysis and Review of Animal Data from Experimental Studies tool to screen studies for inclusion or exclusion. Data extraction and statistical calculations were performed in Microsoft Excel and Stata. Graphical results were created using the ggplot2 package within $\mathrm{R}$ and Microsoft Excel. 


\section{RESULTS}

\section{Publication identification}

Electronic and manual searching retrieved 1737 publications, 469 of which were duplicates found from more than one database search. 1268 publications were screened to determine if they met inclusion criteria, 1179 of these were excluded. Reasons for exclusion included: reviews or non-primary literature, non-SMA, non-genetic therapy intervention, non-rodent model, clinical only data. Of the 89 publications that were deemed relevant, data extraction was completed successfully for 51 [21-71]. The remaining 38 were excluded due to missing, incalculable or irrelevant data. From the included publications, 155 individual comparisons were used in statistical analysis, corresponding to 2573 animals in total. This information is summarised in Fig. 1, with characteristics of included publications in Table 1. Figure 2 shows the distribution of publications and individual comparisons across the years, highlighting a large increase from 2009 onwards.

$N=23$ publications contained comparisons using an oligonucleotide-based approach, $n=26$ used a viral vector and $n=2$ used a combination of both. Genetic therapy agents were delivered between P1 and P25 into a range of models including Taiwanese ( $n=14$ studies) and SMN $\Delta 7$ ( $n=28$ studies) mice, via local (intrathecal, intramuscular and intracranial), systemic (intravascular, subcutaneous and intraperitoneal), or multiple routes of administration. The different characteristics of each study provided the basis of the stratified meta-analysis.

On pooling the 155 comparisons in meta-analysis, we found SMA animals treated with a genetic therapy to survive over 3 times as long as controls (MSR: $3.23,95 \%$ Cl 2.75-3.79; $x^{2}=$ 2671.65, df $=154 ; P<0.0073)$.

\section{Stratification of data}

Type of genetic therapy. Three categories of genetic therapy agents were compared; oligonucleotide-based approaches including antisense oligonucleotides (ASO), peptide morpholinos and naked DNA/RNA, viral vector-based approaches including AAV, adenoviral and lentiviral vectors and oligonucleotide plus viral vector combinatorial approaches. Oligonucleotide-based approaches led to the development of Spinraza, whilst viral vector-based approaches, specifically $A A V$, led to the development of Zolgensma. Therefore, this allows direct comparison of the efficacy of two drugs' rationale, and how successfully these

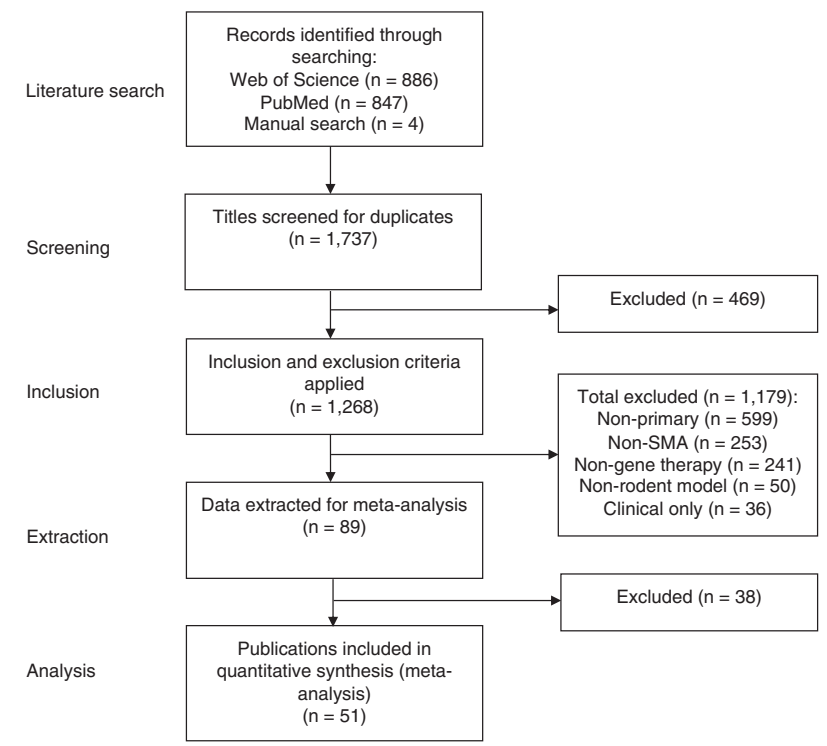

Fig. 1 Flow chart illustrating steps in study identification and assessment of eligibility for inclusion in the meta-analysis. $n$ number of studies. translated to human clinical trials.

All three types of genetic therapy were associated with a significant increase in median survival $\left(X^{2}=38.54, \mathrm{df}=2 ; P<\right.$ 0.0073). Oligonucleotide approaches showed just over three-fold survival advantage (MSR: $3.18,95 \% \mathrm{Cl} 2.58-3.93 ; n$ comparisons $=$ 85; Fig. 3A) whilst viral vector approaches provided a similar increase (MSR: $3.33,95 \% \mathrm{Cl} 2.60-4.27 ; n=66$; Fig. 3A). Efficacy was very similar, if slightly increased, when oligonucleotide and viral vectors were combined within a single treatment (MSR: 3.41, 95\% $\mathrm{Cl} 0.89-13.08 ; n=3$; Fig. $3 \mathrm{~A})$. However, only two publications $[70,71]$, containing three individual comparisons, used a combinatorial treatment so efficacy may be overestimated.

Viral vector dosage. Within the different types of genetic therapy, an attempt was made to further stratify by dosage of genetic therapy agent. This was possible for those studies that used viral vector-based approaches as raw data presented in total vector genomes per mouse, or vector genomes $(\mathrm{vg}) / \mathrm{kg}$ could be delineated into discreet groups by conversion of all to $\mathrm{vg} / \mathrm{kg}$. However, dosage delineation for oligonucleotide-based approaches was not possible due to disparity in the presentation of dose. Some publications presented dose as weight-based measures (either absolute $\mu \mathrm{g}$ or $\mu \mathrm{g} / \mathrm{g}$ ) or in molar concentrations. Therefore, only sub-stratification by viral vector dosage is shown here.

Of the 69 comparisons that administered viral vectors, alone or in combination with an oligonucleotide, significant differences between efficacy were observed $\left(x^{2}=1817.93, \mathrm{df}=2 ; P<0.0073\right)$. A minority of comparisons used $\leq \mathrm{e} 12 \mathrm{vg} / \mathrm{kg}$ leading to a small, but significant increase in survival (MSR: $1.29,95 \% \mathrm{Cl} 1.14-1.45 ; n=5$; Fig. 3B). These comparisons either used $\leq \mathrm{e} 12 \mathrm{vg} / \mathrm{kg}$ as a low dose comparison to others in e13 or $\geq e 14 \mathrm{vg} / \mathrm{kg}$ categories, or vector titre may have been limited due to the nature of virus used, as in the case of lentiviral vectors [45] and adenoviral vectors [44]. Approximately equal numbers of comparisons implemented either e13 or e14 vg/kg viral dosages. e13 vg/ $\mathrm{kg}$ was associated with the largest survival advantage (MSR: 4.83, 95\% Cl 3.32-7.03; $n=30$; Fig. 3B). Finally, the highest dose of viral vector ( $\geq e 14 \mathrm{vg} /$ $\mathrm{kg}$ ) produced a larger increase in survival than $\leq \mathrm{e} 12 \mathrm{vg} / \mathrm{kg}$, but not as high as e13 vg/kg (MSR: $2.72,95 \% \mathrm{Cl} 1.98-3.74 ; n=34 ;$ Fig. 3B).

Therapeutic target. Since SMA is a monogenic disease, augmentation of SMN protein production has been the preferred genetic therapy strategy, however SMN-dependent, SMN-independent and SMN-plus strategies have been reported in the literature, with differing improvements in median survival $\left(x^{2}=363.02, \mathrm{df}=2\right.$; $P<0.0073)$. Augmentation of SMN protein, whether this be through replacement of the SMN1 gene, or manipulation of SMN2 splicing, provided the largest survival benefit here and was used in $86 \%$ of comparisons included (MSR: $3.65,95 \% \mathrm{Cl}$ 3.08-4.34; $n=134$; Fig. 4A). A smaller number of comparisons addressed non-SMN targets: Uba1, Plastin3, PTEN, IGF1, CT1, Stathmin, Stasimon, Myostatin and Synaptotagmin13. These led to a more modest increase in survival (MSR: 1.30, 95\% Cl 1.15-1.47; $n=17$; Fig. 4A). Furthermore, when combining SMN-dependent and -independent targets into an SMN-plus strategy, the lifespan of animals fell between that of each constituent therapy (MSR: 2.98, 95\% Cl 1.06-8.36; $n=4$; Fig. 4A). However, only 72 animals were treated in this manner in three publications.

When directly comparing $S M N$-dependent therapeutic targets $\left(X^{2}=507.97, \mathrm{df}=1 ; P<0.0073\right)$ it was seen that SMN1-targeted therapies produced an MSR of $4.47(95 \% \mathrm{Cl} 3.34-5.97 ; n=43$; Fig. 4B) compared to SMN2-dependent MSR of $3.36(95 \% \mathrm{Cl}$ 2.73-4.14; $n=90$; Fig. 4B).

Mouse model. Although the search employed in this review aimed to retrieve studies from any SMA rodent species, every publication used a mouse model. Most commonly, the SMN $\Delta 7$ 
Table 1. Overview of pre-clinical gene therapy applications in SMA mouse models.

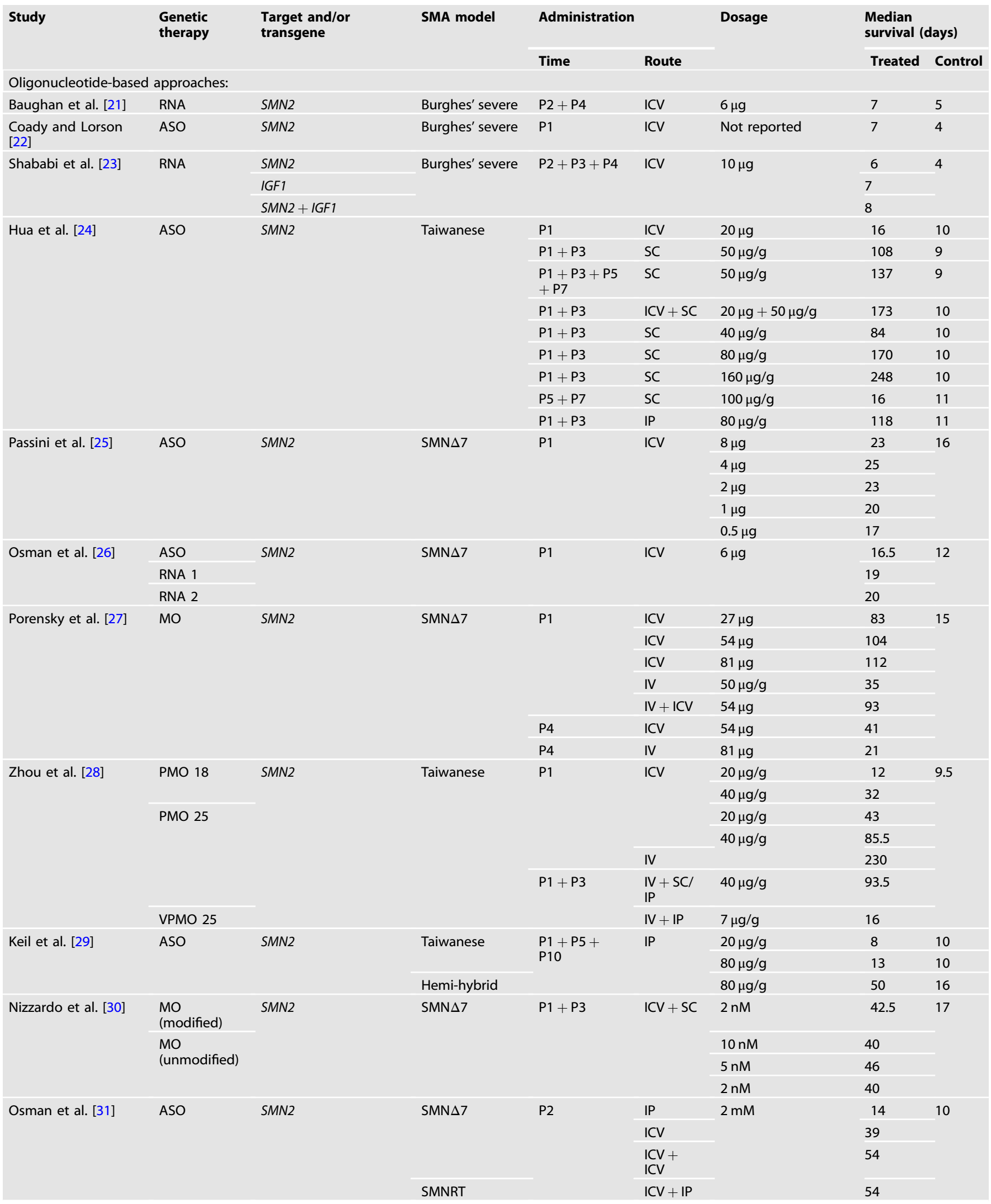


Table 1 continued

\begin{tabular}{|c|c|c|c|c|c|c|c|c|}
\hline Study & $\begin{array}{l}\text { Genetic } \\
\text { therapy }\end{array}$ & $\begin{array}{l}\text { Target and/or } \\
\text { transgene }\end{array}$ & SMA model & \multicolumn{2}{|c|}{ Administration } & Dosage & \multicolumn{2}{|c|}{$\begin{array}{l}\text { Median } \\
\text { survival (days) }\end{array}$} \\
\hline & & & & & ICV & & 175 & 17 \\
\hline Bogdanik et al. [32] & & & & P25 & & & 100 & \\
\hline \multirow[t]{2}{*}{ Hua et al. [33] } & ASO & SMN2 & Taiwanese & $\mathrm{P} 1+\mathrm{P} 3$ & SC & $120 \mathrm{mg} / \mathrm{kg}$ & 237 & 10 \\
\hline & & & & & $\mathrm{ICV}+\mathrm{SC}$ & $\begin{array}{l}120 \mathrm{mg} / \mathrm{kg}+30 \\
\mu \mathrm{g} \text { decoy }\end{array}$ & 212 & 10 \\
\hline \multirow{5}{*}{ Zhou et al. [34] } & & & & & SC & & 261 & \\
\hline & & & & & ICV & $20 \mu \mathrm{g} / \mathrm{g}$ & 43 & \\
\hline & & & & & SC & & 58 & \\
\hline & & & & & ICV & $10 \mu \mathrm{g} / \mathrm{g}$ & 22 & \\
\hline & & & & & SC & & 25 & \\
\hline Lin et al. [38] & ASO & SMN2 & Taiwanese & P1 & SC & $80 \mu \mathrm{g} / \mathrm{g}$ & 19.7 & 7.7 \\
\hline \multirow[t]{10}{*}{ Osman et al. [39] } & ASO E1 MO & SMN2 & $\mathrm{SMN} \Delta 7$ & P1 & ICV & $2 \mu \mathrm{l}$ of $40 \mathrm{nM}$ & 47.8 & 12.3 \\
\hline & ASO E1 $1^{\text {MOv1 }}$ & & & & & & 15.8 & \\
\hline & ASO E1 $1^{\text {MOv2 }}$ & & & & & & 10.2 & \\
\hline & ASO E1 $1^{\text {MOv3 }}$ & & & & & & 19 & \\
\hline & ASO E1 $1^{\mathrm{MOv} 4}$ & & & & & & 19.5 & \\
\hline & ASO E1 $1^{\text {MOv5 }}$ & & & & & & 15.3 & \\
\hline & ASO E1 $1^{\text {MOv6 }}$ & & & & & & 18.8 & \\
\hline & ASO E1 ${ }^{\mathrm{MOv} 7}$ & & & & & & 15.8 & \\
\hline & ASO E1 ${ }^{\text {MOv8 }}$ & & & & & & 18.3 & \\
\hline & ASO E1 ${ }^{\text {MOv9 }}$ & & & & & & 17.5 & \\
\hline $\begin{array}{l}\text { Shabanpoor et al. } \\
\text { [42] }\end{array}$ & $\begin{array}{l}\text { PMO (Br- } \\
\text { ApoE) }\end{array}$ & & & & & & 78 & \\
\hline \multirow[t]{3}{*}{ d'Ydewalle et al. [43] } & ASO & SMN2 & SMN $\Delta 7$ & $\mathrm{P} 1+\mathrm{P} 3$ & SC & $400 \mathrm{mg} / \mathrm{kg}$ & 18 & 18 \\
\hline & SSO & & & & & $50 \mathrm{mg} / \mathrm{kg}$ & 25 & \\
\hline & $\mathrm{ASO}+\mathrm{SSO}$ & & & & & $\begin{array}{l}400 \mathrm{mg} / \mathrm{kg}+50 \mathrm{mg} / \\
\mathrm{kg}\end{array}$ & 37 & \\
\hline \multicolumn{9}{|c|}{ Viral vector-based approaches: } \\
\hline Lesbordes et al. [44] & Ad & $C T 1$ & $\begin{array}{l}\text { NSE-Cre+ } \\
\text { Smn }\end{array}$ & P5-7 & IM & $10 \mathrm{e} 8 \mathrm{pfu} / \mathrm{mouse}$ & 44.4 & 33.7 \\
\hline Azzouz et al. [45] & EIAV SIN LV & SMN1 & $\mathrm{SMN} \Delta 7$ & P2 & IM & $1.2 \mathrm{e} 8 \mathrm{vg} / \mathrm{mouse}$ & 18 & 13 \\
\hline \multirow[t]{2}{*}{ Passini et al. [46] } & SSAAV8 & SMN1 & SMN $\Delta 7$ & P1 & $I C V+I S$ & $5 e 10 \mathrm{vg} / \mathrm{mouse}$ & 50 & 15 \\
\hline & SCAAV8 & & & & & $1.7 \mathrm{e} 10 \mathrm{vg} / \mathrm{mouse}$ & 157 & 16 \\
\hline Valori et al. [47] & ScAAV9 & Codon optimised SMN1 & $\mathrm{SMN} \Delta 7$ & P1 & IV & $10 \mathrm{e} 11 \mathrm{vg} / \mathrm{mouse}$ & 69.1 & 11.2 \\
\hline Foust et al. [48] & ScAAV9 & SMN1 & $\mathrm{SMN} \Delta 7$ & P1 & IV & $5 e 11 \mathrm{vg} /$ mouse & 250 & 15.5 \\
\hline $\begin{array}{l}\text { Dominguez et al. } \\
\text { [49] }\end{array}$ & ScAAV9 & SMN1 & $\operatorname{SMN} \Delta 7$ & P1 & IV & $4.5 \mathrm{e} 10 \mathrm{vg} / \mathrm{mouse}$ & 160 & 13.7 \\
\hline \multirow[t]{2}{*}{ Glascock et al. [50] } & scAAV9 & SMN1 & Burghes' severe & P1 & ICV & $2 \mathrm{e} 11 \mathrm{vg} / \mathrm{mouse}$ & 17 & 7 \\
\hline & & & & & IV & & 10 & \\
\hline \multirow[t]{2}{*}{ Glascock et al. [51] } & scAAV9 & SMN1 & $\mathrm{SMN} \Delta 7$ & P2 & IV & $2 \mathrm{e} 10 \mathrm{vg} / \mathrm{mouse}$ & 34.9 & 11 \\
\hline & & & & $\mathrm{P} 2+\mathrm{P} 3$ & ICV & & 126.7 & \\
\hline \multirow{2}{*}{ Shababi et al. [52] } & scAAV9 & SMN1 & SMN $\Delta 7$ & P2 & IV & $1 \mathrm{e} 11 \mathrm{vg} /$ mouse & 23.5 & 12 \\
\hline & scAAV9 & Codon optimised SMN1 & SMN $\Delta 7$ & $\mathrm{P} 1+\mathrm{P} 2$ & & $5 e 13 \mathrm{vg} / \mathrm{kg}$ & 26 & 12 \\
\hline
\end{tabular}




\begin{tabular}{|c|c|c|c|c|c|c|c|c|}
\hline \multirow[t]{2}{*}{ Study } & \multirow[t]{2}{*}{$\begin{array}{l}\text { Genetic } \\
\text { therapy }\end{array}$} & \multirow[t]{2}{*}{$\begin{array}{l}\text { Target and/or } \\
\text { transgene }\end{array}$} & \multirow[t]{2}{*}{ SMA model } & \multicolumn{2}{|l|}{ Administration } & \multirow[t]{2}{*}{ Dosage } & \multicolumn{2}{|c|}{$\begin{array}{l}\text { Median } \\
\text { survival (days) }\end{array}$} \\
\hline & & & & Time & Route & & Treated & Control \\
\hline \multirow[t]{2}{*}{$\begin{array}{l}\text { Benkhelifa-Ziyyat } \\
\text { et al. [53] }\end{array}$} & & & & & $\begin{array}{l}\text { IM } \\
\text { (2 limbs) }\end{array}$ & & & \\
\hline & & & & & $\begin{array}{l}\text { IM } \\
\text { (4 limbs) }\end{array}$ & & 163 & \\
\hline Tsai et al. [54] & AAV1 & IGF1 & Burghes' severe & P1 & IV & $3.4 \mathrm{e} 9 \mathrm{vg} / \mathrm{mouse}$ & 12 & 9 \\
\hline \multirow[t]{3}{*}{ Passini et al. [55] } & scAAV9 & SMN1 & SMN $\Delta 7$ & $\mathrm{P} 1$ & $\mathrm{ICV}+\mathrm{IT}$ & $5 \mathrm{e} 10 \mathrm{vg} / \mathrm{mouse}$ & 153 & 17 \\
\hline & & & & & & $1 \mathrm{e} 10 \mathrm{vg} / \mathrm{mouse}$ & 70 & \\
\hline & & & & & & $1 \mathrm{e} 9 \mathrm{vg} / \mathrm{mouse}$ & 18 & \\
\hline \multirow[t]{7}{*}{ Robbins et al. [56] } & scAAV9 & SMN1 & $\mathrm{SMN} \Delta 7$ & $\mathrm{P} 2$ & ICV & $1 \mathrm{e} 11 \mathrm{vg} / \mathrm{mouse}$ & 204 & 14 \\
\hline & & & & P3 & & & 75 & \\
\hline & & & & P4 & & & 167 & \\
\hline & & & & P5 & & & 37 & \\
\hline & & & & P6 & & & 34 & \\
\hline & & & & P7 & & & 28 & \\
\hline & & & & P8 & & & 18 & \\
\hline Little et al. [57] & ScAAV9 & PTEN & SMN $\Delta 7$ & P1 & IV & $10 \mathrm{e} 10 \mathrm{vg} /$ mouse & 23.5 & 10 \\
\hline Powis et al. [58] & ssAAV9 & Uba1 & Taiwanese & P1 & IV & $2.4 \mathrm{e} 11 \mathrm{vg} / \mathrm{mouse}$ & 12 & 9 \\
\hline \multirow[t]{5}{*}{ Odermatt et al. [59] } & scAAV9 & SMN2 via U7-ESE-B & SMN $\Delta 7$ & $\mathrm{P} 1+\mathrm{P} 2$ & ICV & $4.07 \mathrm{e} 12 \mathrm{vg} / \mathrm{kg}$ & 22 & 12 \\
\hline & & & & & & $1.75 \mathrm{e} 13 \mathrm{vg} / \mathrm{kg}$ & 25.5 & \\
\hline & & & & & & $3.21 \mathrm{e} 13 \mathrm{vg} / \mathrm{kg}$ & 33 & \\
\hline & & & & & & $4.34 \mathrm{e} 13 \mathrm{vg} / \mathrm{kg}$ & 34 & \\
\hline & & & & & & $2.27 \mathrm{e} 14 \mathrm{vg} / \mathrm{kg}$ & 195 & \\
\hline \multirow[t]{6}{*}{ Armbruster et al. [60] } & scAAV9 & Codon optimised SMN1 & SMN $\Delta 7$ & $\mathrm{P} 1$ & ICV & $1.9 \mathrm{e} 13 \mathrm{vg} / \mathrm{kg}$ & 201 & 16 \\
\hline & & & & & & $3 e 13 \mathrm{vg} / \mathrm{kg}$ & 346 & \\
\hline & & & & & & $7.5 \mathrm{e} 13 \mathrm{vg} / \mathrm{kg}$ & 154 & \\
\hline & & & & & & $1.9 \mathrm{e} 13 \mathrm{vg} / \mathrm{kg}$ & 283 & \\
\hline & & & & & $I C V+I V$ & $3 e 13 \mathrm{vg} / \mathrm{kg}$ & 188 & \\
\hline & & & & & & $7.5 \mathrm{e} 13 \mathrm{vg} / \mathrm{kg}$ & 262 & \\
\hline Alrafiah et al. [61] & ssAAV9 & Plastin3 & SMN $\Delta 7$ & $\mathrm{P} 1$ & IT & $5 e 10 \mathrm{vg} /$ mouse & 17.5 & 12.5 \\
\hline Villalon et al. [62] & scAAV9 & Stathmin 1 & Smn2B/- & $\mathrm{P} 2$ & ICV & $1 \mathrm{e} 11 \mathrm{vg} /$ mouse & 27.02 & 19.04 \\
\hline \multirow[t]{3}{*}{ Donadon et al. [63] } & AAV9 & SMN2 via ExSpeU1s & Taiwanese & $\mathrm{P} 1+\mathrm{P} 3$ & IP & $1.5 \mathrm{e} 12 \mathrm{vg} / \mathrm{mouse}$ & 219 & 10 \\
\hline & & & & $\mathrm{P} 1$ & & & 150 & \\
\hline & & & & $\mathrm{P} 1+\mathrm{P} 3$ & & $1.5 \mathrm{e} 11 \mathrm{vg} / \mathrm{mouse}$ & 13.56 & \\
\hline \multirow{2}{*}{$\begin{array}{l}\text { Rashnonejad et al. } \\
\text { [64] }\end{array}$} & ssAAV9 & SMN1 & SMN $\Delta 7$ & E14-15 & ICV & $4 \mathrm{e} 10 \mathrm{vg} / \mathrm{mouse}$ & 63 & 12 \\
\hline & scAAV9 & & & & & & 105 & \\
\hline Simon et al. [65] & scAAV9 & Stasimon & SMN $\Delta 7$ & $\mathrm{P} 1$ & ICV & $1 \mathrm{e} 11 \mathrm{vg} / \mathrm{mouse}$ & 15.03 & 14.12 \\
\hline \multirow[t]{8}{*}{ Osman et al. [66] } & scAAV9 & SMN1 & $\mathrm{SMN} \Delta 7$ & $\mathrm{P} 2$ & ICV & $1 \mathrm{e} 11 \mathrm{vg} / \mathrm{mouse}$ & 70 & 10 \\
\hline & & D. rerio Smn & & & & & 70 & \\
\hline & & X. laevis Smn & & & & & 38 & \\
\hline & & D. melanogaster Smn & & & & & 13 & \\
\hline & & C. elegans Smn & & & & & 11 & \\
\hline & & S. pombe Smn & & & & & 9 & \\
\hline & & $S M N \_236$ & & & & & 15 & 13 \\
\hline & & & Smn2B/- & & & & 36 & 25 \\
\hline \multirow[t]{3}{*}{ Ahlskog et al. [67] } & scAAV8 & Klf15 & Taiwanese & $\mathrm{P} 1$ & IV & $2 \mathrm{e} 10 \mathrm{vg} / \mathrm{mouse}$ & 13.8 & 12.82 \\
\hline & & & & & & $1 \mathrm{e} 10 \mathrm{vg} / \mathrm{mouse}$ & 7.88 & \\
\hline & & & Smn2B/- & & & $2 \mathrm{e} 10 \mathrm{vg} / \mathrm{mouse}$ & 21.73 & 20.7 \\
\hline \multirow[t]{4}{*}{ Besse et al. [68] } & AAV9 & Codon optimised SMN1 & SMN $\Delta 7$ & $\mathrm{P} 1$ & ICV & $4.5 \mathrm{e} 10 \mathrm{vg} / \mathrm{mouse}$ & 15.5 & 16 \\
\hline & & $(h S Y N)$ & & & & $1.2 \mathrm{e} 11 \mathrm{vg} / \mathrm{mouse}$ & 39.5 & \\
\hline & & Codon optimised SMN1 & & & & $4.5 \mathrm{e} 10 \mathrm{vg} / \mathrm{mouse}$ & 221 & \\
\hline & & $(h P G K)$ & & & IV & & 142 & \\
\hline
\end{tabular}


Table 1 continued

\begin{tabular}{|c|c|c|c|c|c|c|c|c|}
\hline Study & $\begin{array}{l}\text { Genetic } \\
\text { therapy }\end{array}$ & $\begin{array}{l}\text { Target and/or } \\
\text { transgene }\end{array}$ & SMA model & \multicolumn{2}{|c|}{ Administration } & Dosage & \multicolumn{2}{|c|}{$\begin{array}{l}\text { Median } \\
\text { survival (days) }\end{array}$} \\
\hline Nizzardo et al. [69] & AAV9 & Syt13 & $\mathrm{SMN} \Delta 7$ & P1 & IM & $5 e 10 \mathrm{vg} / \mathrm{mouse}$ & 18 & 12 \\
\hline \multirow[t]{6}{*}{ Kaifer et al. [70] } & ScAAV9 & Plastin3 & \multirow[t]{3}{*}{$\mathrm{SMN} \Delta 7$} & \multirow[t]{6}{*}{ P1 } & IV & $1 \mathrm{e} 11 \mathrm{vg} / \mathrm{mouse}$ & 15 & 15 \\
\hline & ASO & Plastin3 & & & ICV & $1 \mathrm{nmol}$ & 17 & 15 \\
\hline & $\begin{array}{l}\text { ASO }+ \\
\text { scAAV9 }\end{array}$ & SMN2 + Plastin3 & & & $\mathrm{ICV}+\mathrm{IV}$ & $\begin{array}{l}1 \mathrm{nmol}+1 \mathrm{e} 11 \mathrm{vg} / \\
\text { mouse }\end{array}$ & 14 & 15 \\
\hline & $\begin{array}{l}\text { ASO }+ \\
\text { SCAAV9 }\end{array}$ & SMN2 + Plastin3 & \multirow[t]{3}{*}{ Smn2B/- } & & $\mathrm{ICV}+\mathrm{IV}$ & $\begin{array}{l}2 \mathrm{nmol}+1 \mathrm{e} 11 \mathrm{vg} / \\
\text { mouse }\end{array}$ & 43.5 & 17 \\
\hline & scAAV9 & Plastin3 & & & IV & $1 \mathrm{e} 11 \mathrm{vg} / \mathrm{mouse}$ & 43.75 & 30 \\
\hline & ScAAV9 & Plastin3 & & & IV & $3 e 11 \mathrm{vg} /$ mouse & 45 & 30 \\
\hline Zhou et al. [71] & AAV & Myostatin & Taiwanese & P1 & SC & $2.5 \mathrm{e} 10 \mathrm{vg} / \mathrm{mouse}$ & 12 & 10 \\
\hline
\end{tabular}

ASO antisense oligonucleotide, $M O$ morpholino, $P M O$ peptide morpholino, SSO splice switching oligonucleotide, AAV adeno-associated viral vector, ss single astranded, sc self-complementary, EIAV equine infectious anaemia virus, SIN self-inactivating, LV lentiviral vector, IV intravascular, IT intrathecal, ICV intracerebral ventricular, IS intraspinal, IM intramuscular, IP intraperitoneal, SC subcutaneous, P1 post-natal day $1, v g$ vector genomes, pfu plaque forming unit.

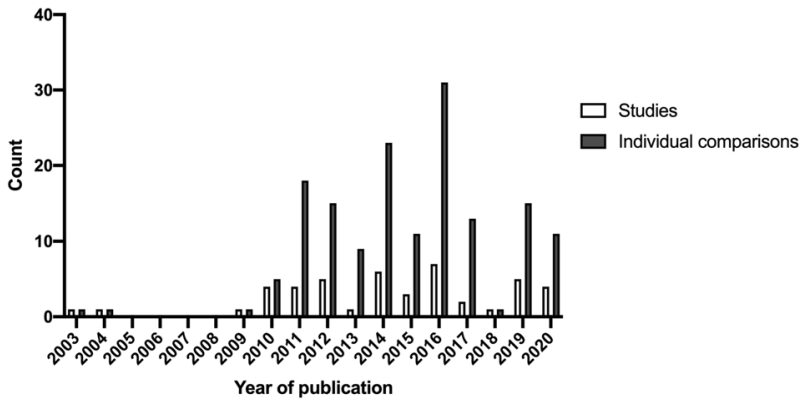

Fig. 2 Distribution of studies and the individual comparisons they contain. 51 eligible studies were included in this meta-analysis. Some publications contained multiple comparisons within the main study; together 155 individual comparisons were assessed here.

model was used, followed by the severe Taiwanese model. Other mouse models were also used but in lower frequencies, so have been grouped into one category here. These other models included Smn2B/- $(n=5)$, type II/III Burgheron $(n=2)$, hemihybrid $(n=1)$, Burghes' severe $(n=8)$, SMNRT $(n=1)$, moderate type II $\left(S m n^{+/-} S M N 2 S M N \Delta 7, n=1\right)$, neuronal Smn deletion (NSE$\left.\mathrm{Cre}+\mathrm{Smn}^{F 7 / F 7}, n=1\right)$. Improvements in median survival differed between mouse model sub-strata $\left(x^{2}=471.05, \mathrm{df}=2 ; P<0.0073\right)$.

Taiwanese mice provide the most severe phenotype within the pure groupings in this review, on average surviving up to 15 days [72]. When genetic therapy was administered to Taiwanese mice a more than five-fold improvement in median survival was found (MSR: $5.49,95 \% \mathrm{Cl} 3.83-7.87 ; n=41$; Fig. 5 ). SMN $\Delta 7$ mice survive 15-22 days [73] without therapeutic intervention, so are useful when a slightly longer lifespan may reveal more subtle phenotypic benefits of a therapy. SMN $\Delta 7$ mice showed a 2.9 -fold increase in survival (MSR: 2.92, 95\% Cl 2.45-3.49; $n=96$; Fig. 5). Less frequently used mice models showed a more modest increase in survival (MSR: 1.65, 95\% Cl 1.28-2.12; $n=18$; Fig. 5).

Route of administration. Both local (intracranial, intrathecal and intramuscular) and systemic (intravascular, intraperitoneal and

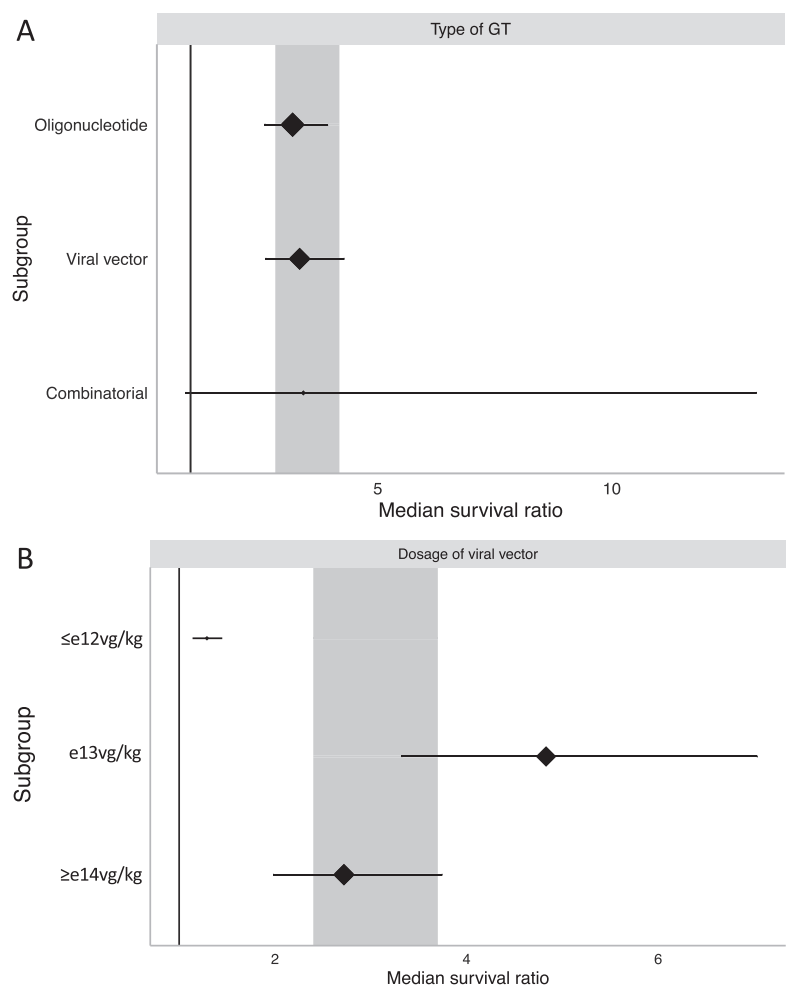

Fig. 3 Stratification by type of gene therapy and dosage of viral vector. Both (A) type of gene therapy and (B) dosage of viral vector accounted for significant heterogeneity in median survival ratio (MSR $P<0.0073$ ). B Sub-strata were defined as viral vector dosage of $\leq \mathrm{e} 12, \mathrm{e} 13$, and $\geq \mathrm{e} 14 \mathrm{vg} / \mathrm{kg}$. A, B Plots represent mean $\pm 95 \%$ confidence intervals with the size of diamonds representing the number of comparisons within each stratum. The vertical line at $M S R=1$ represents a neutral treatment effect. Grey rectangles represent global 95\% confidence intervals. 

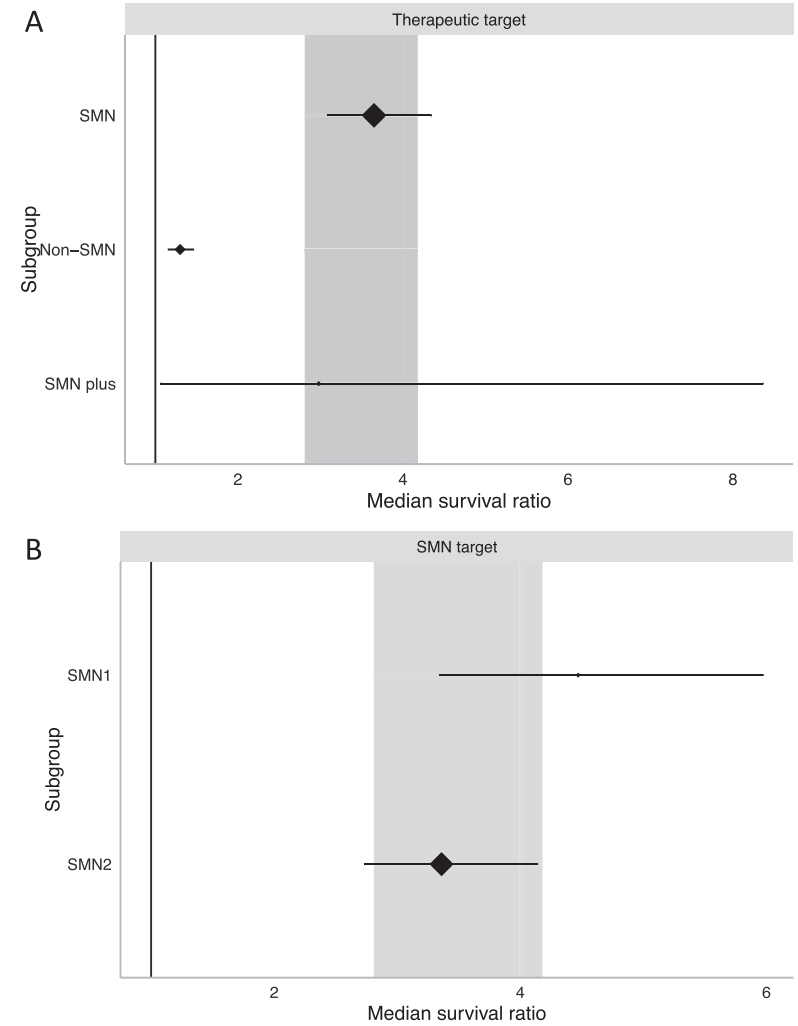

Fig. 4 Stratification by therapeutic target. Significant differences in MSR are apparent dependent on $(\mathbf{A})$ overall therapeutic target (MSR $P<0.0073$ ) and between (B) SMN1- and SMN2-dependent therapies (MSR $P<0.0073$ ). Plot represents mean $\pm 95 \%$ confidence intervals with the size of diamonds representing the number of comparisons within each stratum. The vertical line at $M S R=1$ represents a neutral treatment effect. Grey rectangle represents global $95 \%$ confidence intervals.

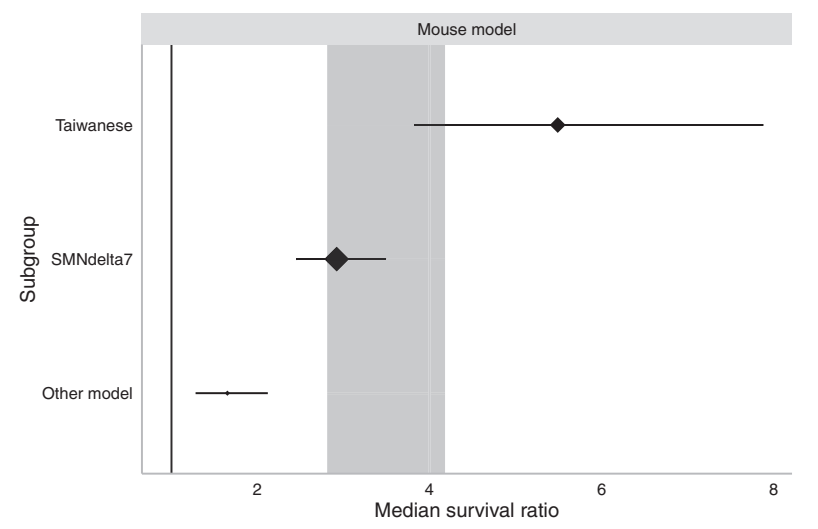

Fig. 5 Stratification by SMA mouse model. Significant differences were found between mouse models (MSR $P<0.0073$ ). Plots show mean $\pm 95 \%$ confidence intervals with the size of diamonds representing the number of comparisons within each stratum. The vertical line at $M S R=1$ represents a neutral treatment effect. Grey rectangle represents global $95 \%$ confidence intervals.

subcutaneous) routes of administration were reported in the literature, but significant differences in efficacy were observed between these routes $\left(x^{2}=422.34, \mathrm{df}=5 ; \quad P<0.0073\right)$. Despite accumulating evidence supporting the systemic nature of SMA, local routes of administration continue being used often (Fig. 6A).

CNS delivery of therapeutics by either intracranial or intrathecal

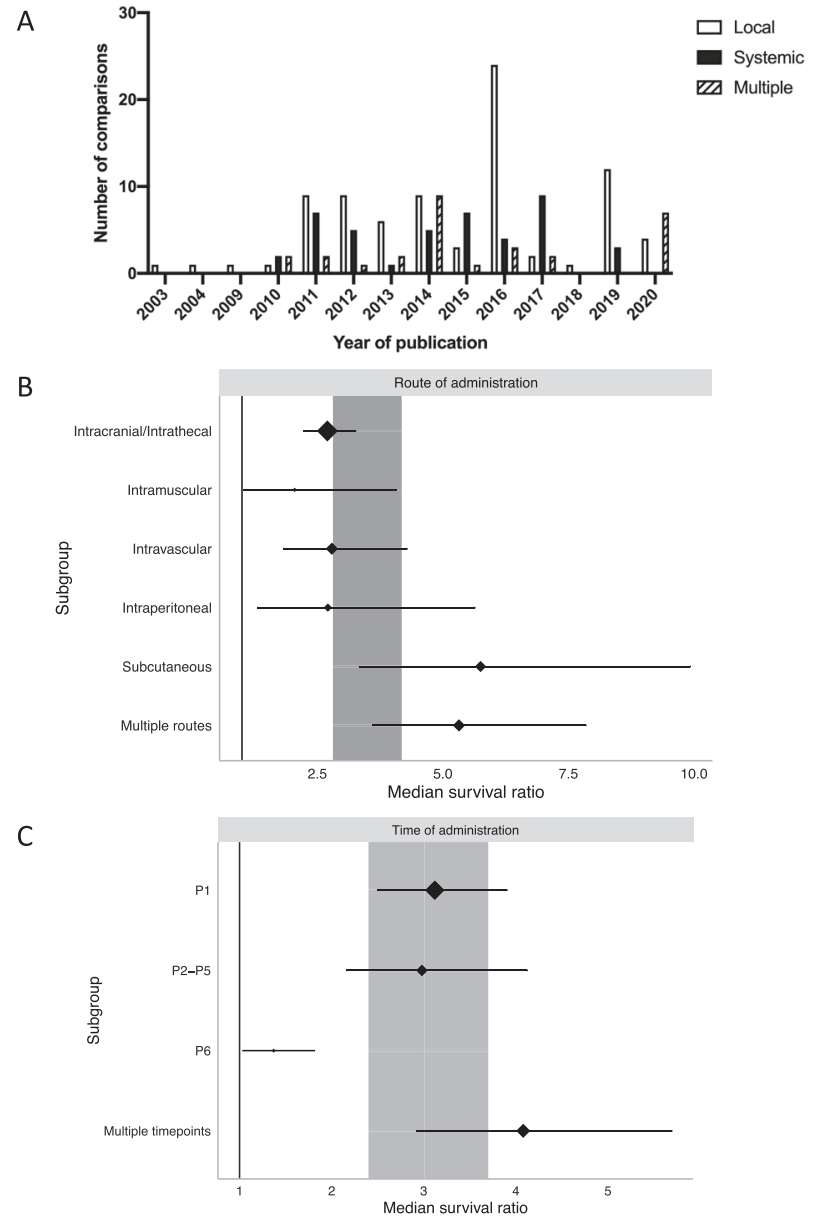

Fig. 6 Stratification by route and time of administration of gene therapy. A Stratification by year of publication and delivery route shows that local administration remains an often used strategy despite the systemic nature of SMA. B, C Forest plots demonstrating significant differences in survival data within both route and time of administration strata (MSR $P<0.0073$ ). Plots represent mean $\pm 95 \%$ confidence intervals with the size of diamonds representing the number of comparisons within each stratum. The vertical line at $M S R=1$ in B, C represents a neutral treatment effect. Grey rectangles represent global $95 \%$ confidence intervals.

injection was the most commonly used route of administration and was associated with an almost three-fold increase in survival (MSR: 2.70, 95\% Cl 2.22-3.27; $n=77$; Fig. 6B). Local, intramuscular delivery more than doubled the lifespan of treated mice (MSR: $2.05,95 \% \mathrm{Cl}$ $1.03-4.07 ; n=6$; Fig. $6 \mathrm{~B}$ ), highlighting the importance of treating the muscular pathology of SMA. Regarding systemic routes, both intravascular (MSR: 2.79, 95\% Cl 1.82-4.28; $n=22$; Fig. 6B) and intraperitoneal (MSR: $2.71,95 \% \mathrm{Cl} 1.30-5.63 ; n=10$; Fig. 6B) delivery were associated with similar survival rates as CNS and intramuscular delivery. Subcutaneous delivery was the third systemic route addressed, providing the largest MSR $(5.75,95 \% \mathrm{Cl} 3.33-9.92 ; n=$ 18; Fig. 6B). Finally, $14 \%$ of comparisons investigated used multiple routes of administration within their study. In most cases, these comparisons used intracranial injection in combination with a second route. This led to a 5.32 -fold increase in survival $(95 \% \mathrm{Cl}$ $3.60-7.84 ; n=22$; Fig. $6 \mathrm{~B}$ ).

Time of administration. SMA in its most severe form is a childhood disease with onset in utero. Therefore, early intervention is thought to be key to halting disease progression or providing phenotypic benefit before irreversible pathology occurs [56]. Here, the time of 
Table 2. Multivariate meta-regression analysis.

\begin{tabular}{|c|c|c|c|}
\hline Comparison & Variable(s) included & Variable(s) excluded & Multivariate $P$ value \\
\hline \multirow[t]{3}{*}{ Type of genetic therapy } & & Oligonucleotide & Not analysed \\
\hline & & Viral vector & \\
\hline & & Combinatorial & \\
\hline \multirow[t]{3}{*}{ Viral vector dosage } & $\leq \mathrm{e} 12 \mathrm{vg} / \mathrm{kg}$ & & 0.552 \\
\hline & $\mathrm{e} 13 \mathrm{vg} / \mathrm{kg}$ & & \\
\hline & $\geq e 14 \mathrm{vg} / \mathrm{kg}$ & & \\
\hline Therapeutic target & SMN-independent & & \\
\hline \multirow[t]{2}{*}{ SMN-dependent target } & & SMN1 & Not analysed \\
\hline & & SMN2 & \\
\hline \multirow[t]{2}{*}{ Mouse model } & $\mathrm{SMN} \Delta 7$ & Taiwanese & 0.5691 \\
\hline & Other & & \\
\hline Route of administration & Multiple & & \\
\hline \multirow[t]{3}{*}{ Time of administration } & P1 & & 0.1757 \\
\hline & P2-P5 & & \\
\hline & $\geq \mathrm{P} 6$ & & \\
\hline
\end{tabular}

Multivariate meta-regression revealed a large degree of collinearity within data, leading to only 69 out of 155 individual comparisons being included in the analysis. Variables that were dropped from the analysis due to this collinearity are shown here. Following exclusion, only therapeutic target was significantly associated with increased survival ( $P=0.0019 ;$ SMN-dependent MSR: $5.71,95 \% \mathrm{Cl} 3.54-9.23 ; n=134 ;$ SMN-independent MSR: $1.28,95 \% \mathrm{Cl} 0.82-2.01 ; n=17)$.

genetic therapy administration greatly impacted the resulting efficacy $\left(x^{2}=284.93, \mathrm{df}=3 ; \quad P<0.0073\right)$. The need for early intervention is highlighted here with approximately half of comparisons administering genetic therapy on the day of birth. Intervention on P1 leads to a 3.12-fold increased lifespan $(95 \% \mathrm{Cl}$ 2.49-3.90; $n=83$; Fig. 6C). Slightly later intervention within the P2-P5 window provided similar results (MSR: 2.98, 95\% Cl 2.16-4.12; $n=24$; Fig. 6C). Administration at P6 or later provided a much lesser, yet still significant benefit (MSR: $1.37,95 \% \mathrm{Cl} 1.03-1.82 ; n=6$; Fig. 6C). Finally, repeated administrations provide the largest increase in survival time seen (MSR: $4.08,95 \% \mathrm{Cl} 2.92-5.69 ; n=39$; Fig. 6C).

Post-hoc meta-regression. The above stratified univariate analysis was implemented to identify patterns within data that may suggest aspects of experimental design that lead to the largest survival extensions. However, stratified univariate analyses do not allow for assessment of how variables interact. Therefore at the suggestion of reviewers we added a multivariate metaregressionin an attempt to identify sources of covariance. We consider this part of our analysis post-hoc and this should be appreciated when interpreting these results.

On proceeding to multivariate meta-regression, there was a significant reduction in the number of studies that could be included due to collinearity (only 69 out of 155 individual comparisons included). In all experimental variables assessed, except time of administration and viral vector dosage, at least one category was dropped from the analysis due to this collinearity (Table 2). When comparing the type of genetic therapy used, none of the oligonucleotide-, viral vector-based or combinatorial approaches could be analysed. Within other variables, Taiwanese mice, intramuscular delivery and SMN-plus therapeutic targets could not be analysed, as well as a complete removal of the SMN1and SMN2-specific analysis (Table 2).
When the remaining variables were analysed, the only variable found to be significantly associated with survival outcome was gene target $(P=0.0019)$. SMN-dependent therapies led to an MSR of 5.71 $(95 \% \mathrm{Cl} 3.54-9.23 ; n=134)$, whilst SMN-independent targets had an MSR of $1.28(95 \% \mathrm{Cl} 0.82-2.01 ; n=17)$. Otherwise, the model did not suggest a predictive effect of any other variable (Table 2).

In summary, only a small amount of information can be learned from the multivariate meta-regression, other than the fact that there is indeed a large degree of collinearity within this dataset, as is expected in pre-clinical literature.

Publication bias. Publication bias in meta-analyses can occur due to unintentional exclusion of missing data, potentially causing misinformed conclusions to be drawn. Evidence of publication bias can be sought using funnel plots, Egger's regression and trim and fill analyses (Fig. 7). While there was no obvious asymmetry to the funnel plot, only a small relative number of comparisons reported an effect size $<1(n=7$; Fig. 7A). On Egger's regression we found a positive intercept (Fig. 7B), suggesting the presence of an excess of small, imprecise comparisons overstating efficacy in this analysis. Trim and fill analysis did not suggest the presence of any 'missing' publications. However, Trim and fill analysis has been described as a relatively insensitive technique and can be an inadequate method of correcting for publication bias [74].

Translation of pre-clinical data to clinical trials. One aim of this systematic review and meta-analysis was to assess how predictive pre-clinical studies can be when translated to clinical trials. Therefore, here we have reviewed $n=6$ clinical trials; $n=5$ of which were assessing Spinraza efficacy [75-79] and $n=1$ assessing Zolgensma [10]. Table 3 details data presented in these studies. Unfortunately, meta-analytic techniques could not be applied to these data for two reasons [1]: no consistent outcomes were reported in all six trials, highlighting the need for consistent 

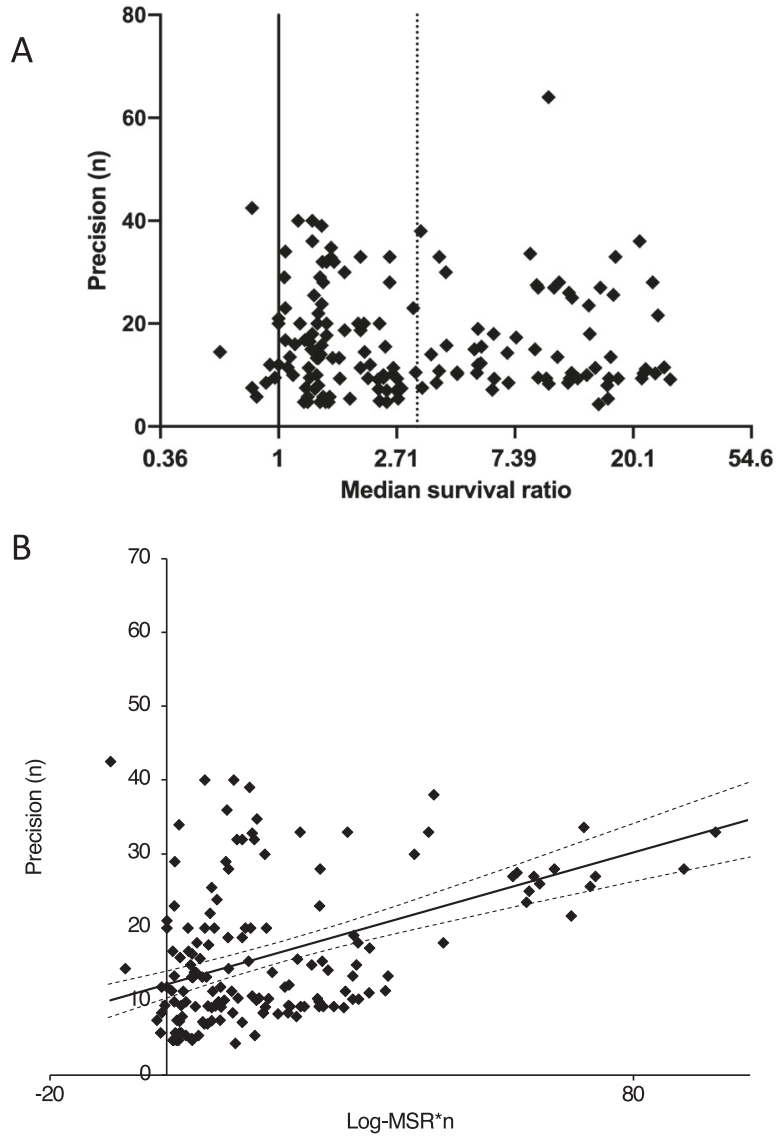

Fig. 7 Publication bias in included publications. A Funnel plot showing untransformed median survival against study precision (number of animals), with no apparent asymmetry found. B Egger's regression (solid line) revealed positive intercept suggesting imprecise studies showed overstated efficacy. Dotted lines represent $95 \%$ confidence intervals.

outcome reporting across clinical trials, allowing direct comparison of data; and [2] only two out of six studies included control groups [78, 79], meaning statistical methods could not be employed.

Overall, 255 affected people were treated and 83 control individuals were included across the 6 studies. 16 deaths were reported in treatment vs 39 deaths in control groups. Serious adverse events were reported in all studies except NCT01780246 and NCT01494701 [75]. In the studies that reported HFMSE (Hammersmith Functional Motor Scale Expanded) scores, some treated patients showed increases by $\geq 3$ points, which are said to be clinically meaningful. HINE-2 (Hammersmith Infant Neurological Examination section 2) and CHOP-INTEND (Children's Hospital of Philadelphia Infant Test of Neuromuscular Disorders) scores also increased following treatment. Half of the studies reported motor milestones in treated patients compared to control groups, all showing improved responses in treatment groups.

\section{DISCUSSION}

In this meta-analysis of 51 publications, containing data from 2573 animals, we found that overall, genetic therapies led to approximately a three-fold increase in median survival. Stratified meta-analysis suggested a significant impact of type of therapy, mouse models, time and route of administration on perceived treatment effect. To our knowledge, this is the first quantitative meta-analysis of published literature of genetic therapy for SMA.
Two other systematic reviews [80, 81] were found, but neither analysed survival benefits. Van der Bent et al. [80] assessed ASO use in heritable neurodegenerative or neuromuscular disorders, including SMA, however, the only data quantitatively analysed pertained to Duchenne Muscular Dystrophy. Qomi et al. [81] systematically describe the development of multiple SMA therapeutic advances at both pre-clinical and clinical level.

With two genetic therapy agents approved for the treatment of SMA patients, a major question concerns the predictive value of pre-clinical studies of oligonucleotide-based approaches that led to Spinraza and viral vector-based approaches that led to Zolgensma. The mechanism and efficacy of Spinraza have been extensively reviewed elsewhere (see refs. [82-85]). A recent, succinct review [86] of pre-clinical AAV9 gene therapy for SMA highlights multiple animal models, including large animals and non-human primates (NHPs).

We observed a significant improvement of median survival with the use of both oligonucleotide- and viral vector-based approaches, with very similar resulting MSRs (3.33 and 3.18, respectively) in the pre-clinical studies analysed in this manuscript. A recent paper [87] compared the Zolgensma NCT02122952 [10] and Spinraza ENDEAR NCT02193074 [78] clinical trials and found that patients treated with Zolgensma had a 20\% higher probability of prevented death, than patients treated with Spinraza (risk ratio $1.2,95 \% \mathrm{Cl} 1.1-1.3$ ). At the last follow up visit in each trial, $100 \%$ of Zolgensma patients were alive, whereas only $84 \%$ of Spinraza patients were [87]. However, several limitations of this comparative study should be noted [88]. Trial design (including aspects such as multi- versus single-centre design) and baseline characteristics of treated patients (including age at first dose, mean disease duration and mean motor function score) were not adjusted for in the number needed to treat analysis conducted in this study and therefore potentially confound any conclusions drawn. Baseline characteristics show a more severe, older patient population in the ENDEAR trial, perhaps explaining the apparent lower efficacy concluded by Dabbous et al. [87]. It should also be stated that the authors of this comparative study were Avexis employees.

With regards to an oligonucleotide plus viral vector combinatorial approach, the efficacy of combinatorial treatment here in fact led to the most pronounced survival benefit, but data are minimal as only two publications $[70,71]$ attempted this. Within the clinical setting, three patients from the Zolgensma NCT02122952 [10] clinical trial are now said to be also being treated with Spinraza, but data from these patients are not available at the time of writing. The phase 4 RESPOND clinical trial has been designed to administer Spinraza to infants previously treated by Zolgensma, who may have responded sub-optimally to the viral vector therapy. Enrollment is due to begin globally in 2021.

Mendell et al. [10] presented data from two cohorts of Zolgensma treatment in their clinical trial; one low dose of $6.7 \times$ $10^{13} \mathrm{vg} / \mathrm{kg}$ and one high dose of $2.0 \times 10^{14} \mathrm{vg} / \mathrm{kg}$. Their rationale for using these two dosages was that in selected pre-clinical models, the low dose doubled survival, but the high dose led to a 250 day survival compared to 15 day control survival [47-49, 89]. In contrast, in the preclinical data (which entailed a broader selection of paradigms), a lower dose (e13 vg/kg; $n=30)$ was in fact associated with greater efficacy than higher ( $\geq e 14 \mathrm{vg} / \mathrm{kg} ; n=34$ ) viral vector dosage. However, there were some differences in experimental design so this finding may be influenced by unaccounted confounders. Respectively, e13 and $\geq e 14 \mathrm{vg} / \mathrm{kg}$ dosage strata showed differences in use of mouse model $(83 \%$ vs $73.5 \%$ : SMN $\triangle 7$ mice), route of delivery ( $43 \%$ vs $58 \%$ : ICV) and transgene (10\% vs $5.9 \%$ : codon-optimised SMN1). Raw median survival ranges of treated SMA animals also differ between the two strata (e13 vg/kg: 7.9-346 days; $\geq e 14 \mathrm{vg} / \mathrm{kg}$ : 9-250 days) in favour of the e13 vg/kg dosage. Biologically, it may be possible that 
Table 3. Characteristics of clinical trials using Spinraza and Zolgensma.

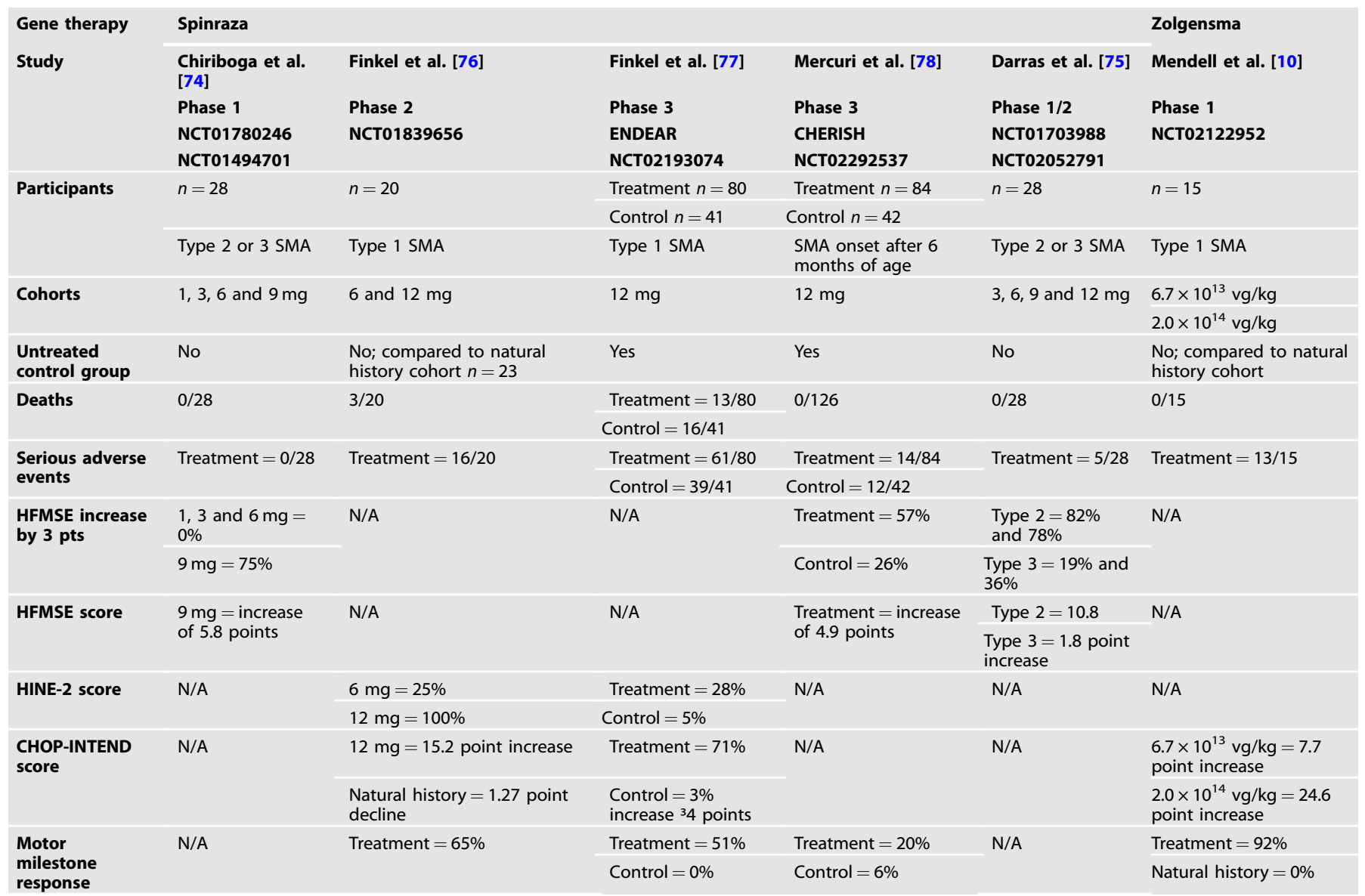

Outcome measures reported at set follow up time points: Chiriboga et al. [75]: 9-14 months, Finkel et al. [77]: up to 32 months, Finkel et al. [78]: day 394, Mercuri et al. [79]: 15 months, Darras et al. [76]: days 253 and 1050, respectively, Mendell et al. [10]: 24 months. HFMSE, HINE-2, and CHOP-INTEND scores represent change from baseline. Finkel et al. [77] define motor milestone response as "improvement of two or more levels per motor milestone category in at least one category". Finkel et al. [78] define motor milestone response as "improvement in at least one HINE-2 motor milestone with more categories with improvement than worsening". Mercuri et al. [79] define motor milestone response as achievement of " $\geq 1$ new World Health Organisation motor milestone".

transgene saturation had occurred in the higher dose. Potentially, if SMN protein was already produced at supraphysiological levels at e13 vg/kg, as suggested within Passini et al. $[46,55]$, BenkhelifaZiyyat et al. [53], and Dominguez et al. [49], increasing viral vector dosage beyond this in rodent models may not lead to a further increase in survival and perhaps be even less efficacious. It has also recently been shown that supraphysiological levels of SMN leads to a late-onset gain of toxic function phenotype caused by disrupted snRNP biogenesis and neuroinflammatory-linked transcriptome changes [90].

It is also important to note the potential safety concerns over high-dose AAV vector therapies. A recent report has highlighted that $34 \%$ of Zolgensma-treated patients across five clinical trials, a managed access programme and commercial use suffered some degree of hepatotoxicity [91]. Subacute liver failure has been reported in at least two cases of high-dose Zolgensma-treated patients $\left(6.25 \times 10^{14}\right.$ and $11.55 \times 10^{14}$ total vector genomes) [92]. Not limited to the treatment of SMA, two children enrolled in the ASPIRO clinical trial for X-linked myotubular myopathy have recently died of sepsis following AAV8_AT-132 therapy [93]. Both boys were in the high-dose cohort administering $3 \times 10^{14} \mathrm{vg} / \mathrm{kg}$, which equates to a dose in excess of $4 \times 10^{15}$ total vector genomes [94]. Bearing these concerns in mind, it is crucial to design strategies that mitigate these risks and investigate aspects of vector design that could negate the need for such high viral loads.
Pre-clinical assessment of therapeutic efficacy can be heavily influenced by the disease model in which the therapy is applied. For SMA, many mouse models exist with varying phenotypes ranging from severe to more mild phenotypes mimicking type II or III SMA. Although SMA mouse models are the most commonly used, models from other species are also available. Increasingly, more non-mouse studies are appearing in the literature using zebrafish [58, 95, 96], cats [97], pigs [98] and NHPs [48, 99]. However, these were not included in this review in order to appreciate the effects of study design and quality more reliably.

Here, we observed greater survival benefits when genetic therapy was given to Taiwanese mice than in treated SMN $\Delta 7$ mice. Furthermore, bimodal survival curves were reported in at least three comparisons assessing AAV-treated $\mathrm{SMN} \Delta 7$ mice, perhaps suggesting there is a population of animals whose phenotype cannot be ameliorated by AAV-mediated therapeutics. In these publications, the first group of animals died before $\sim 1$ month of age (17-27 days [46], 25-35 days [51] and 27-32 days [49]). The four seminal papers [46-49] first describing AAV_SMN-mediated increase in survival all used SMN $\Delta 7$ mice. These papers cited the choice of SMN $\Delta 7$ mice due to the robust phenotype including an approximate 2-week lifespan, loss of MNss, skeletal muscle atrophy and progressive body weight decline. The SMN $\Delta 7$ model had also widely been used in previous pharmacological efficacy studies due to this phenotype [47]. 
It is possible that the mix of different SMA severities within the collated group of less frequently used mouse models contributed to the lower survival benefit seen. For example, Burghes' severe mice survive 4-6 days on average, whilst type II/III models may survive into adulthood. We did not attempt to delineate a severe and a mild group from these other models to avoid inflicting bias when categorising less frequently used models.

The manner in which a therapy is delivered is important to both patients and clinicians. Spinraza is delivered through an intrathecal injection, whilst Zolgensma is intravenously administered. Lumbar puncture in young children, such as those under the age of 6 months with type I SMA, especially those with severely distorted spines, can be distressing and has associated risks not seen with other modes of delivery. Mercuri et al. [79] observed $9 \%$ of adverse events were associated with lumbar puncture $24 \mathrm{~h}$ post Spinraza delivery, rising to $15 \%$ at $168 \mathrm{~h}$ and these were at least $5 \%$ higher than in the sham lumbar puncture control group. Intrathecal drug delivery, in bypassing the blood-brain barrier, provides good CNS penetrance. SMN protein levels augmented by Spinraza are restored in anterior horn cells, but all tissues outside of the CNS are unaffected. Similarly, restricting SMN production to neurons through transcriptional targeting with the synapsin promoter in AAV9 led to reduced rescue in the SMN $\Delta 7$ mouse model ([68]; this study was excluded from our meta-analysis due to being outside the cut-off date). CNS-targeted therapies may improve survival of SMA patients by preventing MN degeneration and its consequences, but hitherto masked peripheral organ damage may become increasingly prevalent in the clinical phenotype, presenting yet unknown burdens. Because of this issue, systemic gene delivery has been a point of interest within recent SMA research.

The definition of a therapeutic window in which administration of a therapeutic agent provides clinical benefit is important, particularly in a disease like severe SMA whose genesis is in utero. Studies have aimed to define this window [56]. For some time, it has been thought that the pre-clinical therapeutic window for SMA exists from the day of birth to $\sim 3$ days afterwards. Recently an AAV9_SMN therapy for SMA has been delivered to mice in utero for the first time, with results indicating a significant increase in survival compared to untreated animals [64], highlighting the potential of fetal genetic therapy for SMA too. Here, similar efficacies can be seen when genetic therapy was administered on the day of birth, or between P2 and P5 (MSR: 3.12 and 2.98, respectively). Pre-symptomatic delivery of treatment may prevent development of the SMA phenotype and the irreversible damage that accompanies this, perhaps due to the deficiency being corrected during the period of neuromuscular junction maturation [4]. Later delivery, on or after P6, causes a dramatic decrease in efficacy (MSR: 1.37), consistent with current knowledge that symptom onset begins at approximately this time, such as reduction in body weight from P6 onwards in SMN $\Delta 7$ mice [47]. Administering repeated doses of genetic therapy increased MSR further compared to the leading single time point (P1). Of the comparisons that administered genetic therapy at multiple time points, $55 \%$ of these used an oligonucleotide approach. This is consistent with the delivery of Spinraza in the clinic, where intrathecal injections are given every four months during the stable dosing phase, in contrast to a single dose of Zolgensma.

Monogenic diseases such as SMA are prime candidates for gene replacement therapies, thus it is not surprising that $86 \%$ of comparisons reviewed here used an $S M N$-dependent approach and these were associated with the greatest survival improvements. It is also reasonable that replacement of the missing SMN1 gene would provide more benefit than augmentation of SMN protein produced by targeting SMN2, as we have identified via a 1.3-fold difference between MSRs (4.47 and 3.36, respectively). Nevertheless, the contributions of disease modifiers are increasingly being linked with the alteration of SMA phenotypes. Plastin3 and NCALD are protective modifiers of SMA in humans, although further modifiers have been found in animals [100]. When studying the interactome of SMN and SMA disease modifiers, non-SMN proteins have been discovered as potential therapeutic targets. Non-SMN targets have been reviewed excellently elsewhere $[3,101]$. Within this meta-analysis 17 comparisons targeted non-SMN proteins with a $44 \%$ increase in survival, albeit lower than SMN-dependent survival.

Further evidence for the use of non-SMN targets to treat SMA is available from non-genetic therapy clinical trials formerly evaluating Olesoxime (now discontinued) and currently assessing Reldesemtiv and SRK-015. These drugs aim to combat oxidative stress in mitochondria, muscle fatigue and improve muscle strength, respectively. With regards to addressing both SMN and non-SMN targets, also known as a SMN plus strategy, it is possible to use ASOs alone [23], viral vectors alone or both [70], to express or modify each target. Here, these approaches led to an MSR of 2.98, higher than that of non-SMN-dependent strategies. Many further publications were found during the literature search using SMN plus strategies, but were ineligible to be included as they modified the non-SMN target via germline transgenesis, instead of gene therapy delivered in vivo. Two example studies showed promising results with transgenic animals $\left(\mathrm{Smn}^{-1-} \mathrm{SMN} 2^{\mathrm{tg} / 0}\right.$ Chp $1^{\text {vac/wt }}$ ) plus SMN2 targeting ASOs [100] as well as transgenic animals (Smn ${ }^{-1-}$ SMN2 KLF15 Mtg) plus viral vectors [102]. A further publication reported administration of scAAV9_DOK7, a neuromuscular junction protein, to $S m n 2 B /-$ mice, leading to a significant increase in median survival by 1 day, however, this was not included in the meta-analysis due to being identified after the pre-specified search cut-off date [103].

Limitations of meta-analytic statistics are, of course, present. Risk of bias was prevalent in a random sample of publications describing in vivo research [104]; coupled with a proclivity for the "file drawer problem", selective publication of positive results, published treatment efficacies are generally inflated. As conventional meta-analytic techniques could not be used with median survival data, an estimate of standard error was made using sample sizes, weights and inter-study variance so that a random effects model could be implemented $[12,13]$. While not as precise as the gold-standard hazard ratio model used in clinical metaanalyses, we believe this model approach to be valid in the context of the limitations in the data. We have tested the same dataset multiple times and have managed the risk of type 1 errors by using Bonferroni correction.

A significant limitation in this meta-analysis is the application of a univariate model, which does not allow for assessment of how variables interact. Given the varied study designs seen in small animal literature, covariance is generally an issue in preclinical meta-analyses. An example from the data presented here highlights this: all but three comparisons administering genetic therapy via subcutaneous delivery used Taiwanese mice as the chosen model. Both of these two sub-strata showed very high MSRs. With a univariate approach it is impossible to determine which of these factors is influential. On this basis, we strongly suggest that these results should be interpreted with caution and considered hypothesis-generating only: resulting questions should be investigated through the conduction of high-quality prospective studies.

Multivariate meta-analysis techniques have been described in preclinical literature $[105,106]$, but their adoption with median survival data has not yet been fully validated. Here, we implemented these techniques in an attempt to identify the variables that most influence survival outcomes following SMA genetic therapy. It is important to note that this was implemented as a post-hoc addition following reviewer comments and was not included in the analysis protocol. However, the results from this analysis were largely inconclusive. Over half of the comparisons included in the original stratified univariate analysis were excluded 
from the multivariate meta-regression due to a high degree of collinearity, meaning that comparison of most variables did all include all possible options presented in the primary literature. The only variable found to be associated with a significant impact on survival outcome was therapeutic target, but again, only SMNdependent and non-SMN therapies could be analysed as SMNplus approaches were excluded; significant exclusions (such as the lack of the Taiwanese model and intramuscular delivery) and low sensitivity of multivariate meta-regression should be borne in mind. Multivariate meta-regressions are known to have a low power to detect associations [107] and since their use with median survival data has not been well studied, we suggest the results of this analysis be interpreted with caution.

With the availability of Spinraza and Zolgensma (and Evrysdi), SMA is the most successfully treated genetic neuromuscular disease. Multiple factors are likely responsible for this: an extensive population of people affected, considerable knowledge of the natural history, a thorough understanding of the genetic basis which has provided various therapeutic strategies, a small cDNA that can be easily packaged, suitable routes of local or systemic delivery, a variety of cellular and animal models for testing, an understanding of the therapeutic window, and the availability of clinical scales or phenotypes that can be measured, among others. The possibility of combinatorial therapy and the existence of a significant pipeline of treatments undergoing pre-clinical and clinical development support further optimism. Not all these factors are relevant to other genetic diseases, but SMA has been a trailblazer and has facilitated the application of similar technology to other CNS diseases. It seems also clear that while Spinraza and Zolgensma improve the SMA phenotype, they are not cures. Further research is therefore necessary to improve therapeutic outcomes in SMA.

\section{CONCLUSIONS}

Genetic therapies have demonstrated therapeutic efficacy for SMA in the clinic. This systematic review and meta-analysis of pre-clinical research has confirmed that genetic therapies can significantly prolong survival, but also that experimental design has a fundamental influence on perceived study outcome. Furthermore, pre-clinical results appear to correlate well with clinical experience of Spinraza and Zolgensma. However, preclinical data are typically at high risk of bias and single paradigms have not reliably predicted translational efficacy. Our conclusions should be borne in mind when conducting further pre-clinical studies of other candidate SMA treatments, as well as more generally small animal research of genetic therapies.

\section{REFERENCES}

1. Lefebvre S, Burgen L, Reboullet S, Clermont $O$, Burlet $P$, Viollet $L$, et al. Identification and characterisation of a spinal muscular atrophy-determining gene. Cell. 1995;80:155-65.

2. Monani UR. Spinal muscular atrophy: a deficiency in a ubiquitous protein; a motor neuron specific disease. Neuron. 2005;48:885-96.

3. Bowerman M, Becker CG, Yáñez-Muñoz RJ, Ning K, Wood M, Gillingwater TH, et al. Therapeutic strategies for spinal muscular atrophy: SMN and beyond. Disease Models Mechanisms. 2017;10:943-54.

4. Farrar MA, Park SB, Vucic S, Carey KA, Turner BJ, Gillingwater TH, et al. Emerging therapies and challenges in spinal muscular atrophy. Ann Neurol. 2017;81:355-68.

5. Martinez-Hernandez R, Soler-Botija C, Also E, Alias L, Caselles L, Gich I, et al. The developmental pattern of myotubes in spinal muscular atrophy indicates prenatal delay of muscle maturation. J Neuropathol Experimental Neurol. 2009;68:474-81.

6. Rudnik-Schoneborn S, Vogelgesang S, Armbrust S, Graul-Neumann L, Fusch C, Zerres K. Digital necroses and vascular thrombosis in severe spinal muscular atrophy. Muscle Nerve. 2010;42:144-7.
7. Wijngaarde CA, Blank AC, Stam M, Wadman RI, van den Berg LH, van der Pol WL. Cardiac pathology in spinal muscular atrophy: a systematic review. Orphanet J Rare Dis. 2017;12:67.

8. Wirth B, Brichta L, Schrank B, Lochmuller H, Blick S, Baasner A, et al. Mildly affected patients with spinal muscular atrophy are partially protected by an increased SMN2 copy number. Hum Genet. 2006;119:422-8.

9. Talbot $\mathrm{K}$, Tizzano EF. The clinical landscape for SMA in a new therapeutic era. Gene Ther. 2017;24:529-33.

10. Mendell JR, Al-Zaidy S, Shell R, Arnold WD, Rodino-Klapac LR, Prior TW, et al. Single-dose gene-replacement therapy for spinal muscular atrophy. N. Eng J Med. 2017;377:1713-22.

11. Baranello G, Darras BT, Day JW, Deconinck N, Klein A, Masson R, et al. Risdiplam in type 1 spinal muscular atrophy. N. Eng J Med. 2021;384:915-23.

12. Vesterinen HM, Sena ES, Egan KJ, Hirst TC, Churolov L, Currie GL, et al. Metaanalysis of data from animal studies: a practical guide. J Neurosci Methods. 2014;221:92-102.

13. Hirst TC, Vesterinen HM, Sena ES, Egan KJ, Macleod MR, Whittle IR. Systematic review and meta-analysis of temozolomide in animal models of glioma: was clinical efficacy predicted? Br J Cancer. 2013;108:64-71.

14. Michiels S, Piedbois P, Burdett S, Syz N, Stewart L, Pignon JP. Meta-analysis when only the median survival times are known: a comparison with individual patient data results. Int J Technol Assess Health Care. 2005;21:119-25.

15. Tierney JF, Stewart LA, Ghersi D, Burdett S, Sydes MR. Practical methods for incorporating summary time-to-event data into meta-analysis. Trials. 2007;8.

16. DerSimonian R, Laird NM. Meta-analysis in clinical trials. Controll Clin Trials. 1986;7:177-88.

17. DerSimonian R, Laird NM. Meta-analysis in clinical trials revisted. Contemperary Clin Trials. 2015;45:139-45.

18. Egger M, Smith GD, Schneider M, Minder C. Bias in meta-analysis detected by a simple, graphical test. Br Med J. 1997;315:629-34.

19. Duval S, Tweedie R. Trim and fill: a simple funnel-plot-based method of testing and adjusting for publication bias in meta-analysis. Biometrics. 2000;56:455-63.

20. Peters JL, Sutton AJ, Jones DR, Abrams KR, Rushton L. Comparison of two methods to detect publication bias in meta-analysis. JAMA. 2006;295:676-80.

21. Baughan TD, Dickson A, Osman EY, Lorson CL. Delivery of bifunctional RNAs that target an intronic repressor and increase SMN levels in an animal model of spinal muscular atrophy. Hum Mol Genet. 2009;18:1600-11.

22. Coady TH, Lorson CL. Trans-splicing-mediated improvement in a severe mouse model of spinal muscular atrophy. J Neurosci. 2010;30:126-30.

23. Shababi M, Glascock J, Lorson C. Combination of SMN trans -splicing and a neurotrophic factor increases the life span and body mass in a severe model of spinal muscular atrophy. Hum Gene Therapy. 2011;22:135-44.

24. Hua Y, Sahashi K, Rigo F, Hung G, Horev G, Bennett CF, et al. Peripheral SMN restoration is essential for long-term rescue of a severe spinal muscular atrophy mouse model. Nature. 2011;478:123-6.

25. Passini MA, Bu J, Richards AM, Kinnecom C, Sardi SP, Stanek LM, et al. Antisense oligonucleotides delivered to the mouse CNS ameliorate symptoms of severe spinal muscular atrophy. Sci Transl Med. 2011;3:72.

26. Osman EY, Yen PF, Lorson CL. Bifunctional RNAs targeting the intronic splicing silencer $\mathrm{N} 1$ increase SMN levels and reduce disease severity in an animal model of spinal muscular atrophy. Mol Ther. 2012;20:119-26.

27. Porensky PN, Mitrpant C, McGovern VL, Bevan AK, Foust KD, Kaspar BK, et al. A single administration of morpholino antisense oligomer rescues spinal muscular atrophy in mouse. Hum Mol Genet. 2012;21:1625-38.

28. Zhou H, Janghra N, Mitrpant C, Dickinson RL, Anthony K, Price $L$, et al. A novel morpholino oligomer targeting ISS-N1 improves rescue of severe spinal muscular atrophy transgenic mice. Hum Gene Ther. 2013;24:331-42.

29. Keil JM, Seo J, Howell MD, Hsu WH, Singh RN, DiDonato CJ. A short antisense oligonucleotide ameliorates symptoms of severe mouse models of spinal muscular atrophy. Mol Ther Nucleic Acids. 2014;3:e174.

30. Nizzardo M, Simone C, Salani S, Ruepp MD, Rizzo F, Ruggieri M, et al. Effect of combined systemic and local morpholino treatment on the spinal muscular atrophy Delta7 mouse model phenotype. Clin Ther. 2014;36:340-56.e5.

31. Osman EY, Miller MR, Robbins KL, Lombardi AM, Atkinson AK, Brehm AJ, et al. Morpholino antisense oligonucleotides targeting intronic repressor Element 1 improve phenotype in SMA mouse models. Hum Mol Genet. 2014;23:4832-45.

32. Bogdanik LP, Osborne MA, Davis C, Martin WP, Austin A, Rigo F, et al. Systemic, postsymptomatic antisense oligonucleotide rescues motor unit maturation delay in a new mouse model for type II/III spinal muscular atrophy. PNAS USA. 2015;112:E5863-72.

33. Hua Y, Liu YH, Sahashi K, Rigo F, Bennett CF, Krainer AR. Motor neuron cellnonautonomous rescue of spinal muscular atrophy phenotypes in mild and severe transgenic mouse models. Genes Dev. 2015;29:288-97.

34. Zhou HY, Meng JH, Marrosu E, Janghra N, Morgan J, Muntoni F. Repeated low doses of morpholino antisense oligomer: an intermediate mouse model of 
spinal muscular atrophy to explore the window of therapeutic response. Hum Mol Genet. 2015;24:6265-77.

35. Olivan S, Calvo AC, Rando A, Herrando-Grabulosa M, Manzano R, Zaragoza P, et al. Neuroprotective Effect of Non-viral Gene Therapy Treatment Based on Tetanus Toxin C-fragment in a Severe Mouse Model of Spinal Muscular Atrophy. Front Mol Neurosci. 2016;9:76.

36. Hammond SM, Hazell G, Shabanpoor F, Saleh AF, Bowerman M, Sleigh JN, et al. Systemic peptide-mediated oligonucleotide therapy improves long-term survival in spinal muscular atrophy. PNAS USA. 2016;113:10962-7.

37. Hosseinibarkooie S, Peters M, Torres-Benito L, Rastetter RH, Hupperich K, Hoffmann A, et al. The power of human protective modifiers: PLS3 and CORO1C unravel impaired endocytosis in spinal muscular atrophy and rescue SMA phenotype. Am J Hum Genet. 2016;99:647-65.

38. Lin TL, Chen TH, Hsu YY, Cheng YH, Juang BT, Jong YJ. Selective Neuromuscular Denervation in Taiwanese Severe SMA Mouse Can Be Reversed by Morpholino Antisense Oligonucleotides. Plos One. 2016;11:e0154723.

39. Osman EY, Washington CW, Kaifer KA, Mazzasette C, Patitucci TN, Florea KM, et al. Optimization of morpholino antisense oligonucleotides targeting the intronic repressor element1 in spinal muscular atrophy. Mol Ther. 2016;24:1592-601.

40. Arnold W, McGovern VL, Sanchez B, Li J, Corlett KM, Kolb SJ, et al. The neuromuscular impact of symptomatic SMN restoration in a mouse model of spinal muscular atrophy. Neurobiol Dis. 2016;87:116-23.

41. Riessland M, Kaczmarek A, Schneider S, Swoboda KJ, Lohr H, Bradler C, et al. Neurocalcin delta suppression protects against spinal muscular atrophy in humans and across species by restoring impaired endocytosis. Am J Hum Genet. 2017;100:297-315.

42. Shabanpoor F, Hammond SM, Abendroth F, Hazell G, Wood MJA, Gait MJ. Identification of a peptide for systemic brain delivery of a morpholino oligonucleotide in mouse models of spinal muscular atrophy. Nucleic Acid Ther. 2017;27:130.

43. d'Ydewalle C, Ramos DM, Pyles NJ, Ng SY, Gorz M, Pilato CM, et al. The antisense transcript SMN-AS1 regulates SMN expression and is a novel therapeutic target for spinal muscular atrophy. Neuron. 2017;93:66-79.

44. Lesbordes JC, Cifuentes-Diaz C, Miroglio A, Joshi V, Bordet T, Kahn A, et al. Therapeutic benefits of cardiotrophin-1 gene transfer in a mouse model of spinal muscular atrophy. Hum Mol Genet. 2003;12:1233-9.

45. Azzouz M, Le T, Ralph GS, Walmsley L, Monani UR, Lee DC, et al. Lentivectormediated SMN replacement in a mouse model of spinal muscular atrophy. J Clin Investig. 2004;114:1726-31.

46. Passini MA, Bu J, Roskelley EM, Richards AM, Sardi SP, O'Riordan CR, et al. CNS targeted gene therapy improves survival and motor function in a mouse model of spinal muscular atrophy. J Clin Investig. 2010;120:1253-64.

47. Valori CF, Ning K, Wyles M, Mead RJ, Grierson AJ, Shaw PJ, et al. Systemic delivery of SCAAV9 expressing SMN prolongs survival in a model of spinal muscular atrophy. Sci Transl Med. 2010;2:35-42.

48. Foust KD, Wang XY, McGovern VL, Braun L, Bevan AK, Haidet AM, et al. Rescue of the spinal muscular atrophy phenotype in a mouse model by early postnatal delivery of SMN. Nature Biotechnology. 2010;28:271-U126.

49. Dominguez E, Marais T, Chatauret N, Benkhelifa-Ziyyat $S$, Duque $S$, Ravassard $P$, et al. Intravenous SCAAV9 delivery of a codon-optimized SMN1 sequence rescues SMA mice. Huma Mol Genet. 2011;20:681-93.

50. Glascock JJ, Osman EY, Wetz MJ, Krogman MM, Shababi M, Lorson CL. Decreasing disease severity in symptomatic, $\operatorname{Smn}(-/-)$;SMN2 $(+/+)$, spinal muscular atrophy mice following scAAV9-SMN delivery. Hum Gene Ther. 2012;23:330-5

51. Glascock JJ, Shababi M, Wetz MJ, Krogman MM, Lorson CL. Direct central nervous system delivery provides enhanced protection following vector mediated gene replacement in a severe model of spinal muscular atrophy. Biochem Biophys Res Commun. 2012;417:376-81.

52. Shababi M, Habibi J, Ma L, Glascock JJ, Sowers JR, Lorson CL. Partial restoration of cardio-vascular defects in a rescued severe model of spinal muscular atrophy. J Mol Cell Cardiol. 2012;52:1074-82.

53. Benkhelifa-Ziyyat S, Besse A, Roda M, Duque S, Astord S, Carcenac R, et al. Intramuscular SCAAV9-SMN injection mediates widespread gene delivery to the spinal cord and decreases disease severity in SMA mice. Mol Ther. 2013;21:282-90.

54. Tsai LK, Chen $\mathrm{CL}$, Ting $\mathrm{CH}$, Lin-Chao $\mathrm{S}$, Hwu WL, Dodge JC, et al. Systemic administration of a recombinant AAV1 vector encoding IGF-1 improves disease manifestations in SMA mice. Mol Ther. 2014;22:1450-9.

55. Passini MA, Bu J, Richards AM, Treleaven CM, Sullivan JA, O'Riordan CR, et al. Translational fidelity of intrathecal delivery of self-complementary AAV9-survival motor neuron 1 for spinal muscular atrophy. Hum Gene Ther. 2014;25:619-30.
56. Robbins KL, Glascock JJ, Osman EY, Miller MR, Lorson CL. Defining the therapeutic window in a severe animal model of spinal muscular atrophy. Hum Mol Genet. 2014;23:4559-68.

57. Little D, Valori CF, Mutsaers CA, Bennett EJ, Wyles $M$, Sharrack B, et al. PTEN depletion decreases disease severity and modestly prolongs survival in a mouse model of spinal muscular atrophy. Mol Ther. 2015;23:270-7.

58. Powis RA, Karyka E, Boyd P, Come J, Jones RA, Zheng $Y$, et al. Systemic restoration of UBA1 ameliorates disease in spinal muscular atrophy. JCI Insight. 2016;1:e87908.

59. Odermatt $P$, Trub J, Furrer L, Fricker R, Marti A, Schumperli D. Somatic Therapy of a Mouse SMA Model with a U7 snRNA Gene Correcting SMN2 Splicing. Mol Ther. 2016;24:1797-805.

60. Armbruster N, Lattanzi A, Jeavons M, Van Wittenberghe L, Gjata B, Marais T, et al. Efficacy and biodistribution analysis of intracerebroventricular administration of an optimized scAAV9-SMN1 vector in a mouse model of spinal muscular atrophy. Mol Ther Methods Clin Dev. 2016;3:16060.

61. Alrafiah A, Karyka E, Coldicott I, Iremonger K, Lewis KE, Ning K, et al. Plastin 3 Promotes Motor Neuron Axonal Growth and Extends Survival in a Mouse Model of Spinal Muscular Atrophy. Mol Ther Methods Clin Dev. 2018;9:81-9.

62. Villalon E, Kline RA, Smith CE, Lorson ZC, Osman EY, O'Day S, et al. AAV9Stathmin 1 gene delivery improves disease phenotype in an intermediate mouse model of spinal muscular atrophy. Hum Mol Genet. 2019;28:3742-54.

63. Donadon I, Bussani E, Riccardi F, Licastro D, Romano G, Pianigiani G, et al. Rescue of spinal muscular atrophy mouse models with AAV9-Exon-specific U1 snRNA. Nucleic Acids Res. 2019;47:7618-32.

64. Rashnonejad A, Chermahini GA, Gunduz C, Onay H, Aykut A, Durmaz B, et al. Fetal gene therapy using a single injection of recombinant AAV9 Rescued SMA Phenotype in Mice. Mol Ther. 2019;27:2123-33.

65. Simon CM, Van Alstyne M, Lotti F, Bianchetti E, Tisdale S, Watterson DM, et al. Stasimon contributes to the loss of sensory synapses and motor neuron death in a mouse model of spinal muscular atrophy. Cell Rep. 2019;29:3885.

66. Osman EY, Bolding MR, Villalon E, Kaifer KA, Lorson ZC, Tisdale S, et al. Functional characterization of SMN evolution in mouse models of SMA. Sci Rep. 2019;9:9472.

67. Ahlskog N, Hayler D, Krueger A, Kubinski S, Claus P, Hammond SM, et al. Muscle overexpression of KIf15 via an AAV8-Spc5-12 construct does not provide benefits in spinal muscular atrophy mice. Gene Ther. 2020;27:505-15.

68. Besse A, Astord S, Marais T, Roda M, Giroux B, Lejeune FX, et al. AAV9-mediated expression of SMN restricted to neurons does not rescue the spinal muscular atrophy phenotype in mice. Mol Ther. 2020;28:1887-901.

69. Nizzardo M, Taiana M, Rizzo F, Benitez JA, Nijssen J, Allodi I, et al. Synaptotagmin 13 is neuroprotective across motor neuron diseases. Acta Neuropathol. 2020;139:837-53.

70. Kaifer KA, Villalon E, Osman EY, Glascock JJ, Arnold LL, Cornelison DDW, et al. Plastin-3 extends survival and reduces severity in mouse models of spinal muscular atrophy. JCI Insight. 2017;2:e89970.

71. Zhou HY, Meng JH, Malerba A, Catapano F, Sintusek P, Jarmin S, et al. Myostatin inhibition in combination with antisense oligonucleotide therapy improves outcomes in spinal muscular atrophy. J Cachexia Sarcopenia Muscle. 2020;11:768-82.

72. Hsieh-Li HM, Chang JG, Jong YJ, Wu MH, Wang NM, Tsai $\mathrm{CH}$, et al. A mouse model for spinal muscular atrophy. Nat Genet. 2000;24:66-70.

73. Le TT, Pham LT, Butchbach MER, Zhang HL, Monani UR, Coovert DD, et al. SMN Delta 7, the major product of the centromeric survival motor neuron (SMN2) gene, extends survival in mice with spinal muscular atrophy and associates with full-length SMN. Hum Mol Genetics. 2005;14:845-57.

74. Simonsohn U, Nelson LD, Simmons JP. p-Curve and Effect Size: Correcting for Publication Bias Using Only Significant Results. Perspectives on psychological science: a journal of the Association for. Psychol Sci. 2014;9:666-81.

75. Chiriboga CA, Swoboda KJ, Darras BT, lannaccone ST, Montes J, De Vivo DC, et al. Results from a phase 1 study of nusinersen (ISIS-SMNRx) in children with spinal muscular atrophy. Neurology. 2016;86:890-7.

76. Darras BT, Chiriboga CA, lannaccone ST, Swoboda KJ, Montes J, Mignon L, et al. Nusinersen in later-onset spinalmuscular atrophy Long-term results from the phase 1/2 studies. Neurology. 2019;92:E2492-E506.

77. Finkel RS, Chiriboga CA, Vajsar J, Day JW, Montes J, De Vivo DC, et al. Treatment of infantile-onset spinal muscular atrophy with nusinersen: a phase 2, openlabel, dose-escalation study. Lancet. 2016;388:3017-26.

78. Finkel RS, Mercuri E, Darras BT, Connolly AM, Kuntz NL, Kirschner J, et al. Nusinersen versus sham control in infantile-onset spinal muscular atrophy. $\mathrm{N}$. Eng J Med. 2017;377:1723-32.

79. Mercuri E, Darras BT, Chiriboga CA, Day JW, Campbell C, Connolly AM, et al. Nusinersen versus sham control in later-onset spinal muscular atrophy. $\mathrm{N}$. Eng J Med. 2018;378:625-35. 
80. van der Bent ML, da Silva OP, van Luijk J, Brock R, Wansink DG. Assisted delivery of antisense therapeutics in animal models of heritable neurodegenerative and neuromuscular disorders: a systematic review and meta-analysis. Sci Rep. 2018;8:4181.

81. Qomi SB, Asghari A, Salmaninejad A, Mojarrad M. Spinal muscular atrophy and common therapeutic advances. Fetal Pediatric Pathol. 2019;38:226-38.

82. Singh NN, Howell MD, Androphy EJ, Singh RN. How the discovery of ISS-N1 led to the first medical therapy for spinal muscular atrophy. Gene Ther. 2017;24:520-6.

83. Singh NN, Lee BM, DiDonato CJ, Singh RN. Mechanistic principles of antisense targets for the treatment of spinal muscular atrophy. Future Med Chem. 2015;7:1793-808.

84. Douglas AG, Wood MJ. Splicing therapy for neuromuscular disease. Mol Cell Neurosci. 2013;56:169-85.

85. Porensky PN, Burghes AHM. Antisense oligonucleotides for the treatment of spinal muscular atrophy. Hum Gene Ther. 2013;24:489-98.

86. Pattali R, Mou YC, Li XJ. AAV9 vector: a novel modality in gene therapy for spinal muscular atrophy. Gene Ther. 2019:26:287-95.

87. Dabbous O, Maru B, Jansen JP, Lorenzi M, Cloutier M, Guerin A, et al. Survival, motor function, and motor milestones: comparison of AVXS-101 relative to nusinersen for the treatment of infants with spinal muscular atrophy type 1. Adv Ther. 2019;36:1164-76.

88. Sandrock AW, Farwell W. Comparisons Between Separately Conducted Clinical Trials: Letter to the Editor Regarding Dabbous O, Maru B, Jansen JP, Lorenzi M, Cloutier M, Guérin A, et al. Adv Ther (2019) 36:1164-76. https://doi.org/10.1007/ s12325-019-00923-8. Ad Ther. 2019;36:2979-81.

89. Foust KD, Nurre E, Montgomery CL, Hernandez A, Chan CM, Kaspar BK. Intravascular AAV9 preferentially targets neonatal neurons and adult astrocytes. Nat Biotechnol. 2009;27:59-65.

90. Van Alstyne M, Tattoli I, Delestree N, Recinos Y, Workman E, Shihabuddin LS, et al. Gain of toxic function by long-term AAV9-mediated SMN overexpression in the sensorimotor circuit. Nat Neurosci. 2021;24:930.

91. Chand D, Mohr F, McMillan H, Tukov FF, Montgomery K, Kleyn A, et al. Hepatotoxicity following administration of onasemnogene abeparvovec (AVXS-101) for the treatment of spinal muscular atrophy. J Hepatol. 2021;74:560-6.

92. Feldman AG, Parsons JA, Dutmer CM, Veerapandiyan A, Hafberg E, Maloney N, et al. Subacute Liver Failure Following Gene Replacement Therapy for Spinal Muscular Atrophy Type 1. J Pediatrics. 2020;225:252.

93. Wilson JM, Flotte TR. Moving Forward After Two Deaths in a Gene Therapy Trial of Myotubular Myopathy. Hum Gene Ther. 2020;31:695-6.

94. Paulk N. Gene therapy: it's time to talk about high-dose AAV. 2020. https://www. genengnews.com/commentary/gene-therapy-its-time-to-talk-about-high-dose-aav/.

95. Hao LT, Burghes AHM, Beattie CE. Generation and Characterization of a genetic zebrafish model of SMA carrying the human SMN2 gene. Mol Neurodegener. 2011;6:24.

96. Hao LT, Duy PQ, Jontes JD, Wolman M, Granato M, Beattie CE. Temporal requirement for SMN in motoneuron development. Hum Mole Genet. 2013;22:2612-25.

97. Duque $S$, Joussemet B, Riviere C, Marais T, Dubreil L, Douar AM, et al. Intravenous administration of self-complementary AAV9 enables transgene delivery to adult motor neurons. Mol Ther. 2009;17:1187-96.

98. Duque SI, Arnold WD, Odermatt P, Li XH, Porensky PN, Schmelzer L, et al. A large animal model of spinal muscular atrophy and correction of phenotype. Ann Neurol. 2015;77:399-414.

99. Meyer K, Ferraiuolo L, Schmelzer L, Braun L, McGovern V, Likhite S, et al. Improving single injection CSF delivery of AAV9-mediated gene therapy for SMA: a dose-response study in mice and nonhuman primates. Mol Ther. 2015;23:477-87.

100. Janzen E, Mendoza-Ferreira N, Hosseinibarkooie S, Schneider S, Hupperich K, Tschanz T, et al. CHP1 reduction ameliorates spinal muscular atrophy pathology by restoring calcineurin activity and endocytosis. Brain. 2018;141:2343-61.

101. Groen EJN, Talbot K, Gillingwater TH. Advances in therapy for spinal muscular atrophy: promises and challenges. Nat Rev Neurol. 2018;14:214-24.

102. Walter LM, Deguise MO, Meijboom KE, Betts CA, Ahlskog N, van Westering TLE, et al. Interventions targeting glucocorticoid-kruppel-like factor 15-branched- chain amino acid signaling improve disease phenotypes in spinal muscular atrophy mice. EBioMedicine. 2018;31:226-42.

103. Kaifer KA, Villalon E, Smith CE, Simon ME, Marquez J, Hopkins AE, et al. AAV9DOK7 gene therapy reduces disease severity in $\mathrm{Smn}(2 \mathrm{~B} /)$ - SMA model mice. Biochem Biophys Res Commun. 2020;530:107-14.

104. Macleod MR, McLean AL, Kyriakopoulou A, Serghiou S, de Wilde A, Sherratt N, et al. Risk of bias in reports of in vivo research: a focus for improvement. Plos Biol. 2015;13:10.

105. Zwetsloot PP, Vegh AMD, Jansen SJ, van Hout GPJ, Currie GL, Sena ES, et al. Cardiac stem cell treatment in myocardial infarction: a systematic review and meta-analysis of preclinical studies. Circ Res. 2016;118:1223-32.

106. Jue TJ, Hirst TC, Sena ES, Macleod MR, McDonald KL. A systematic review and meta-analysis of topoisomerase inhibition in pre-clinical glioma models. NeuroOncology. 2017;19:59.

107. Tanner-Smith EE, Grant S. Meta-Analysis of Complex Interventions. Annual Review of Public Health, 2018; 39:135-51.

\section{AUTHOR CONTRIBUTIONS}

EMC performed data acquisition and statistical analyses. EWM performed the data transformation and visualisation. RJY-M provided conceptual support and interpretation of results. TH performed statistical analyses and provided experience with previous pre-clinical meta-analyses. All authors contributed to manuscript preparation

\section{FUNDING}

This research received no specific grant from any funding agency in the public, commercial or not-for-profit sectors. RJY-M acknowledges general financial support from SMA UK (formerly The SMA Trust), through the UK SMA Research Consortium, for SMA research in his laboratory.

\section{COMPETING INTERESTS}

The authors declare no competing interests.

\section{ADDITIONAL INFORMATION}

Correspondence and requests for materials should be addressed to Ellie M. Chilcott or Rafael J. Yáñez-Muñoz.

Reprints and permission information is available at http://www.nature.com/ reprints

Publisher's note Springer Nature remains neutral with regard to jurisdictional claims in published maps and institutional affiliations.

Open Access This article is licensed under a Creative Commons Attribution 4.0 International License, which permits use, sharing, adaptation, distribution and reproduction in any medium or format, as long as you give appropriate credit to the original author(s) and the source, provide a link to the Creative Commons license, and indicate if changes were made. The images or other third party material in this article are included in the article's Creative Commons license, unless indicated otherwise in a credit line to the material. If material is not included in the article's Creative Commons license and your intended use is not permitted by statutory regulation or exceeds the permitted use, you will need to obtain permission directly from the copyright holder. To view a copy of this license, visit http://creativecommons. org/licenses/by/4.0/.

(c) The Author(s) 2021, corrected publication 2022 